\title{
THE ENUMERATION DEGREES: LOCAL AND GLOBAL STRUCTURAL INTERACTIONS
}

\author{
THEODORE A. SLAMAN AND MARIYA I. SOSKOVA
}

With congratulations to Professor W. Hugh Woodin on the occasion of his sixtieth birthday

\begin{abstract}
We show that the structure of the enumeration degrees $\mathcal{D}_{e}$ has a finite automorphism base consisting of finitely many total elements below the first jump of its least element. As a consequence we obtain that the rigidity of the structure of the enumeration degrees is implied by the rigidity of the local structures of the $\Sigma_{2}^{0}$ enumeration degrees, the $\Delta_{2}^{0}$ Turing degrees and the computably enumerable Turing degrees.
\end{abstract}

\section{INTRODUCTION}

We continue our investigations of the notion of relative definability between sets of natural numbers, presented as a degree structure. There is a spectrum of notions giving a more precise meaning to the term "relative definability". At the two endpoints, the many-one degrees and the hyperarithmetical degrees, we have complete descriptions of the degree structures and these descriptions are completely different.

The partial ordering of the many-one degrees $\mathcal{D}_{m}$ was characterized algebraically by Ershov [4] and Paliutin [11]. It is the unique partial order $P$ with the following properties: it has cardinality the continuum, it is a distributive upper-semi-lattice with least element, it has the countable predecessor property and given any other distributive upper-semi-lattice $L$ with least element, with cardinality less than the continuum, and with the countable predecessor property and given an isomorphism $\pi$ between an ideal $I$ in $L$ and an ideal $\pi(I)$ in $P$, there is an extension $\pi^{*}$ of $\pi$ to an isomorphism between $L$ and $\pi^{*}(L)$, such that $\pi^{*}(L)$ is an ideal in $P$. That is to say that $\mathcal{D}_{m}$ is a universal object in a natural way. Along the same line, the automorphism group of $\mathcal{D}_{m}$ has cardinality $2^{2^{\omega}}$ and every element of $\mathcal{D}_{m}$ other than its least one, $\mathbf{0}_{m}$, has a nontrivial orbit. Consequently, $\mathbf{0}_{m}$ is the only element of $\mathcal{D}_{m}$ which can be defined by its order-theoretic properties.

The partial ordering of the hyperarithmetical degrees $\mathcal{D}_{h}$ was characterized model theoretically by Slaman and Woodin [19]. It is biinterpretable with the structure of second-order arithmetic. There is a way within the ordering $\mathcal{D}_{h}$ to represent the standard model of arithmetic $\langle\mathbb{N},+, *,<, 0,1\rangle$ and each set of natural numbers $X$ so that the relation " $\vec{p}$ represents the set $X \subset \mathbb{N}$ and $\mathbf{x}$ is the hyperarithmetical degree of $X$ " can be defined in $\mathcal{D}_{h}$ as a property of $\vec{p}$ and $\mathbf{x}$. It follows that there is no nontrivial automorphism of $\mathcal{D}_{h}$, it is rigid. Further, a relation

2010 Mathematics Subject Classification. 03D30.

The first author was partially supported by National Science Foundation grant number DMS1301659. The second author was partially supported by an FP7-MC-IOF grant STRIDE (298471) and by Sofia University Science Fund. 
on $\mathcal{D}_{h}$ is order-theoretically definable within $\mathcal{D}_{h}$ if and only if it corresponds to a degree-invariant relation on sets which is definable in second-order arithmetic.

We would like to know where in the spectrum of relative definability rigidity breaks down. However, for the structures that form the middle of this spectrum we have only a partial understanding. Simpson [15] proved that the first order theory of the Turing degrees is computably isomorphic to the theory of secondorder arithmetic. Slaman and Woodin conjectured that the relationship between the structure of the Turing degrees and second-order arithmetic is much stronger. Their biinterpretability conjecture is that $\mathcal{D}_{T}$ can be characterized in the same way as the hyperarithmetical degrees. Slaman and Woodin [19] established that the structure of the Turing degrees has a finite automorphism base and as a consequence obtained that the biinterpretability conjecture is true modulo the use of finitely many parameters. They showed that the automorphism group of the Turing degrees is countable and that every member has an arithmetically definable presentation. Furthermore, they proved that every relation in $\mathcal{D}_{T}$, induced by a degree invariant relation that is definable in second-order arithmetic, is first order definable with parameters in $\mathcal{D}_{T}$. Finally they showed that biinterpretability and rigidity are equivalent for $\mathcal{D}_{T}$.

We will focus on an extension of the Turing degrees, the structure of the enumeration degrees, $\mathcal{D}_{e}$. This structure arises from the relation enumeration reducibility, introduced by Friedberg and Rogers [5] in 1959. Enumeration reducibility is close to Turing reducibility in the spectrum of relative definability. Instead of an effective transformation of computations, it is based on effective transformation of enumerations. Turing reducibility can be expressed in these terms as well: a set $A$ can be computed from a set $B$ if and only if every enumeration of $B \oplus \bar{B}$ can be effectively transformed into an enumeration of $A \oplus \bar{A}$, and so the Turing degrees have a natural isomorphic copy in $\mathcal{D}_{e}$, the total enumeration degrees. Selman [13] showed a reverse connection: every enumeration degree is completely determined by the set of total enumeration degrees above it. Thus the total enumeration degrees are an automorphism base for the enumeration degrees.

The first order theory of this extended structure was characterized by Slaman and Woodin [18] also as computably isomorphic to second-order arithmetic. In contrast to the Turing degrees, where we do not have many examples of natural first order definitions of classes of degrees, in the enumeration degrees we have quite a few. Kalimullin [8] showed that the jump operation is first order definable by a simple formula in the language of partial orders. Papers of Ganchev and Soskova [6] and [7] extended Kalimullin's method to obtain other definability results. This line of investigation culminated in the paper by Cai, Ganchev, Lempp, Miller and Soskova [1] which established the first order definability of the total enumeration degrees. The total enumeration degrees are thus a definable automorphism base for the Turing degrees. This allows us to transfer all results from Slaman and Woodin's automorphism analysis of $\mathcal{D}_{T}$ to $\mathcal{D}_{e}$ : the enumeration degrees have a finite automorphism base, their automorphism group is countable and every member has an arithmetically definable presentation, relations on $\mathcal{D}_{e}$, induced by degree invariant relations that are definable in second-order arithmetic, are definable with parameters in $\mathcal{D}_{e}$, and the rigidity of $\mathcal{D}_{e}$ is equivalent to its 
biinterpretability with second-order arithmetic. Some of these conclusions were obtained earlier by Soskova [20], but the definability of the total degrees provides a more systematic treatment.

We now know that the automorphism problems for the two structures are connected: a nontrivial automorphism of the enumeration degrees induces a nontrivial automorphism of the Turing degrees. Thus the biinterpretability conjecture for the Turing degrees implies biinterpretability of the enumeration degrees and secondorder arithmetic.

In this article we study the complexity of automorphism bases for the enumeration degrees. We combine methods developed in our earlier paper [16] with previously known results to obtain a finite automorphism base of total enumeration degrees below $\mathbf{0}_{e}^{\prime}$. In [16] we investigated the automorphism group of the local substructure of the Turing degrees $\mathcal{D}_{T}\left(\leq \mathbf{0}_{T}^{\prime}\right)$, consisting of the $\Delta_{2}^{0}$ Turing degrees. We established that the local structure of the Turing degrees relates in the same way to first order arithmetic as the Turing degrees and the enumeration degrees relate to second-order arithmetic: it is biinterpretable with first order arithmetic modulo the use of finitely many parameters and that full biinterpretability is equivalent to rigidity for the structure $\mathcal{D}_{T}\left(\leq \mathbf{0}_{T}^{\prime}\right)$. The first order definability of $\mathbf{0}_{e}{ }^{\prime}$ and the total enumeration degrees below $\mathbf{0}_{e}^{\prime}$ now allows us to tie the automorphism problem for the $\Delta_{2}^{0}$ Turing degrees, or equivalently their biinterpretability with first-order arithmetic, to the automorphism problem for the enumeration degrees, or equivalently their biinterpretability with second-order arithmetic: a nontrivial automorphism of $\mathcal{D}_{e}$ yields a nontrivial automorphism of $\mathcal{D}_{T}\left(\leq \mathbf{0}_{T}^{\prime}\right)$. Finally, we connect the rigidity of the enumeration degrees to the rigidity of an even finer structure: the computably enumerable Turing degrees.

\section{Preliminaries}

We start with an overview of the main notions and results that are used in this article. We also give a brief outline of the proof of the main result.

2.1. The enumeration degrees. In this subsection we will introduce enumeration reducibility, its associated degree structure together with certain properties that will be used in this article. For more results about the structure of the enumeration degrees we refer to Cooper [3].

Definition 1 (Friedberg and Rogers [5]). A set $A$ is enumeration reducible to a set $B$ (denoted by $A \leq_{e} B$ ) if there is a c.e. set $\Phi$, such that

$$
A=\Phi(B)=\left\{n: \exists u\left(\langle n, u\rangle \in \Phi \& D_{u} \subseteq B\right)\right\},
$$

where $D_{u}$ denotes the finite set with code (canonical index) $u$ under the standard coding of finite sets.

A set $A$ is enumeration equivalent to a set $B$ (denoted by $A \equiv_{e} B$ ) if $A \leq_{e} B$ and $B \leq_{e} A$. The equivalence class of $A$ under the relation $\equiv_{e}$ is the enumeration degree $d_{e}(A)$ of $A$. The enumeration degrees are ordered by $d_{e}(A) \leq d_{e}(B)$ if and only if $A \leq_{e} B$. The least element in this ordering is $\mathbf{0}_{e}=d_{e}(\emptyset)$, the set of all c.e. sets. We define a least upper bound operation by setting $d_{e}(A) \vee d_{e}(B)=d_{e}(A \oplus B)$. The enumeration jump of a set $A$ was defined by Cooper [2], as $K_{A} \oplus \overline{K_{A}}$, where $K_{A}=\left\{\langle e, x\rangle \mid x \in \Phi_{e}(A)\right\}$. The enumeration jump of the degree of a $A$ is $d_{e}(A)^{\prime}=$ 
$d_{e}\left(K_{A} \oplus \overline{K_{A}}\right)$. Thus the structure of the enumeration degrees $\left\langle\mathcal{D}_{e}, \leq, \vee,^{\prime}, \mathbf{0}_{e}\right\rangle$ is a an upper semi-lattice with least element and jump operation.

We can already state the first of many definability results that will be applied in this article.

Theorem 1 (Kalimullin [8]). The enumeration jump operator is first order definable in $\mathcal{D}_{e}$.

It is not difficult to see that a set $A$ is Turing equivalent to a set $B$ if and only if $A \oplus \bar{A}$ is enumeration equivalent to $B \oplus \bar{B}$. Thus, the map $\iota$, defined by

$$
\iota\left(d_{T}(A)\right)=d_{e}(A \oplus \bar{A}),
$$

is an embedding of $\mathcal{D}_{T}$ in $\mathcal{D}_{e}$, which preserves the order, the least element and the least upper bound. McEvoy [9] showed that it also preserves the jump operation. The image of the Turing degrees under this embedding is the set of total enumeration degrees.

Definition 2. An automorphism base for a structure $\mathcal{A}$ with domain $A$ is a set $B \subseteq A$, such that for very pair of automorphism $\pi_{1}$ and $\pi_{2}$ of $\mathcal{A}$ if $\pi_{1}$ and $\pi_{2}$ agree on all elements in $B$ then $\pi_{1}=\pi_{2}$.

The total enumeration degrees are an important substructure of the enumeration degrees. The following result shows that they are an automorphism base for $\mathcal{D}_{e}$

Theorem 2 (Selman[13]). Let $\mathbf{a}$ and $\mathbf{b}$ be enumeration degrees. Then $\mathbf{a} \leq \mathbf{b}$ if and only if $\{\mathbf{x} \geq \mathbf{b} \mid \mathbf{x}$ is total $\} \subseteq\{\mathbf{x} \geq \mathbf{a} \mid \mathbf{x}$ is total $\}$.

Many properties of the Turing degrees can be transferred to the enumeration degrees via the following result.

Theorem 3 (Cai, Ganchev, Lempp, Miller and Soskova[1]). The set of total enumeration degrees is first order definable in $\mathcal{D}_{e}$.

It follows, in particular, that any automorphism base of the Turing degrees is an automorphism base of the enumeration degrees. Slaman and Woodin [19] show that the set of Turing degrees below $\mathbf{0}^{(5)}$ is an automorphism base for $\mathcal{D}_{T}$. Thus we have the following theorem:

Theorem 4. The set of total enumeration degrees below $\mathbf{0}_{e}^{(5)}$ is an automorphism base for the structure of the enumeration degrees.

A further definability result that we will use in this article concerns the relation "c.e. in" on Turing degrees.

Definition 3. Let $\mathbf{a}$ and $\mathbf{b}$ be Turing degrees. We say that $\mathbf{a}$ is c.e. in $\mathbf{b}$ if there are sets $A \in \mathbf{a}$ and $B \in \mathbf{b}$, such that $A$ is c.e. in $B$.

Theorem 5 (Cai, Ganchev, Lempp, Miller and Soskova [1]). The image of the relation "c.e. in" under the standard embedding of $\mathcal{D}_{T}$ in $\mathcal{D}_{e}$ is first-order definable in the structure of the enumeration degrees.

2.2. The coding method. The structure $\mathcal{D}_{e}\left(\leq \mathbf{0}_{e}^{\prime}\right)$, also called the local structure of the enumeration degrees, consists of the enumeration degrees of $\Sigma_{2}^{0}$ sets. The proof of Theorem 3 extends an earlier result of Ganchev and Soskova [7]: the total enumeration degrees are definable in $\mathcal{D}_{e}\left(\leq \mathbf{0}_{e}^{\prime}\right)$. In [16] we studied the properties 
of the local structure of the Turing degrees $\mathcal{D}_{T}\left(\leq \mathbf{0}_{T}^{\prime}\right)$, which we can view as a definable substructure of $\mathcal{D}_{e}\left(\leq \mathbf{0}_{e}^{\prime}\right)$. We described a method for representing the standard model of first-order arithmetic as a partial order and discussed various ways to define such a structure in $\mathcal{D}_{T}\left(\leq \mathbf{0}_{T}^{\prime}\right)$ with finitely many parameters. In particular we introduced the notion of indexing, which we now transfer to the setting of the enumeration degrees.

Definition 4. We say that $\overrightarrow{\mathbf{p}}$ define an indexing of a sequence $\left\{Z_{i}\right\}_{i<\omega}$, if they define a model of arithmetic $\mathcal{M}$ and a function $\varphi: \mathbb{N}^{\mathcal{M}} \rightarrow \mathcal{D}_{e}$, such that $\varphi\left(i^{\mathcal{M}}\right)=$ $d_{e}\left(Z_{i}\right)$.

Slaman and Woodin [17] showed that in $\mathcal{D}_{T}\left(\leq \mathbf{0}_{T}^{\prime}\right)$ there is an indexing of the c.e. sets which is definable from parameters. Note that the image of the Turing degree $d_{T}\left(W_{e}\right)$ under the standard embedding is the enumeration degree $\iota\left(d_{T}\left(W_{e}\right)\right)=$ $d_{e}\left(\bar{W}_{e}\right)$. In other words, the c.e. Turing degrees are mapped to the $\Pi_{1}^{0}$ enumeration degrees. Transferring Slaman and Woodin's result to the enumeration degrees we have the following:

Theorem 6 (Slaman and Woodin [17]). There are finitely many total parameters in $\mathcal{D}_{e}\left(\leq \mathbf{0}_{e}^{\prime}\right)$ that define an indexing of the sequence $\left\{\bar{W}_{e}\right\}_{e<\omega}$.

An indexing of the sequence $\left\{\bar{W}_{e}\right\}_{e<\omega}$ will be called a basic indexing. Parameters that define a basic indexing will be called basic indexing parameters.

The main result in [16] shows the existence of finitely many parameters in $\mathcal{D}_{T}\left(\leq \mathbf{0}_{T}^{\prime}\right)$ that define an indexing of the $\Delta_{2}^{0}$ sets, i.e. an indexing of the sequence $\left\{X_{e}\right\}_{e<\omega}$, where

- If $\{e\}^{\emptyset^{\prime}}$ is a total $\{0,1\}$-valued function then $X_{e}$ is the set with characteristic function $\{e\}^{\emptyset^{\prime}}$.

- $X_{e}=\emptyset$ otherwise.

Again, we can transfer this result to show that there is indexing definable from finitely many parameters of the sequence $\left\{X_{e} \oplus \overline{X_{e}}\right\}_{e<\omega}$. We will say that this is an indexing of the total $\Delta_{2}^{0}$ sets. In the next section we improve this result to show that every set of basic indexing parameters defines an indexing of the total $\Delta_{2}^{0}$ sets.

Our next goal will be to extend the indexing power of any set of basic indexing parameters outside the local structure. Let us denote by $\mathcal{I} \mathcal{T}\left(\mathbf{0}_{e}^{\prime}\right)$ the union of all intervals $\left[\mathbf{x}, \mathbf{x}^{\prime}\right]$, where $\mathbf{x}$ is a total enumeration degree below $\mathbf{0}_{e}^{\prime}$. The definability of the image of the relation "c.e. in", stated as Theorem 5 , will be one of the main tools used in Section 4, where we show that any set of basic indexing parameters can be used to identify the total enumeration degrees in the set $\mathcal{I T}\left(\mathbf{0}_{e}^{\prime}\right)$. We first extend the indexing power to cover all enumeration degrees that are images of a Turing degree $\mathbf{y}$ that is c.e. in and above some Turing degree $\mathbf{x} \leq_{T} \mathbf{0}^{\prime}$. We show that every set of basic indexing parameters defines a $\mathbf{0}_{e}^{\prime}$-basic indexing: an indexing of the sequence $\left\{Y_{e} \oplus \overline{Y_{e}}\right\}_{e<\omega}$, where $e=\left\langle e_{0}, e_{1}\right\rangle$ and $Y_{e}=X_{e_{1}} \oplus W_{e_{0}}^{X_{e_{1}}}$.

Then relativizing the results from Section 3, we show that every set of basic indexing parameters defines an indexing of the total $\mathcal{I} \mathcal{T}\left(\mathbf{0}_{e}^{\prime}\right)$ sets: an indexing of the sequence $\left\{Z_{e} \oplus \overline{Z_{e}}\right\}_{e<\omega}$, where $e=\left\langle e_{0}, e_{1}\right\rangle$ and:

- If $\left\{e_{0}\right\}^{X_{e_{1}}}$ is a total $\{0,1\}$-valued function then $Z_{e}=X_{e_{1}} \oplus\left\{e_{0}\right\}^{X_{e_{1}}}$. - $Z_{e}=\emptyset$ otherwise.

Finally, in Section 5 we extend the power of any set of basic indexing parameters to cover all total enumeration degrees in the interval $\left[\mathbf{0}_{e}, \mathbf{0}_{\mathbf{e}}^{\prime \prime}\right]$ : we show that they 
define an indexing of the total $\Delta_{3}^{0}$ sets. We can iterate the results from Section 4 and Section 5 in relativized form to cover the total enumeration degrees in the interval $\left[\mathbf{0}_{e}, \mathbf{0}_{e}^{(n)}\right]$ for any natural number $n$. In particular, when $n=5$ we see that any set of basic indexing parameters determines the total enumeration degrees below $\mathbf{0}_{e}^{(5)}$. By Theorem 4 this is an automorphism base for the enumeration degrees.

\section{Step 1: An indexing of the total $\Delta_{2}^{0}$ Sets}

The goal of this section is to prove the following theorem:

Theorem 7. Every set of basic indexing parameters $\vec{p} \in \mathcal{D}_{e}\left(\leq \mathbf{0}_{e}^{\prime}\right)$ defines an indexing of the total $\Delta_{2}^{0}$ sets.

This result is significantly stronger than that from [16], where we showed merely the existence of a finite set of parameters $\vec{p} \in \mathcal{D}_{T}\left(\leq \mathbf{0}_{T}^{\prime}\right)$, defining an indexing of the $\Delta_{2}^{0}$ sets. We will outline the main steps that comprised the proof of the result in [16] and explain how they can be implemented and improved in the context of the enumeration degrees. Using ideas that go back to works of Jockusch and Posner we first showed that every every $\Delta_{2}^{0}$ Turing degree can be determined using four low $\Delta_{2}^{0}$ Turing degrees. Here a degree (Turing or enumeration) is low if its jump is equal to the jump of the least degree. In the enumeration degrees we have an even simpler way of determining the total $\Delta_{2}^{0}$ enumeration degrees from the low enumeration degrees.

Theorem 8 (Ganchev, Soskova [7]). The set of low enumeration degrees is first order definable in $\mathcal{D}_{e}\left(\leq \mathbf{0}_{e}^{\prime}\right)$. Every total enumeration degree $\mathbf{y} \leq \mathbf{0}_{e}^{\prime}$ is the least upper bound of two low enumeration degrees.

Next we showed that every low Turing degree has a unique position with respect to the c.e. Turing degrees and an additional set of $\Delta_{2}^{0}$ Turing degrees $\mathcal{Z}$. The set $\mathcal{Z}$ was constructed in a way that allowed us to argue that there are parameters that index the c.e. sets and a sequence representing $\mathcal{Z}$. Working in the richer world of the enumeration degrees, we show that the image of the c.e. Turing degrees under the standard embedding, the set of $\Pi_{1}^{0}$ enumeration degrees, is sufficient to uniquely determine the position of every low enumeration degree and hence of every total enumeration degree below $\mathbf{0}_{e}^{\prime}$. We prove this in the following two theorems. Recall that a set is d.c.e. if it is the difference of two c.e. sets. The co-d.c.e. sets are the complements of d.c.e. sets and the co-d.c.e. enumeration degrees are those that contain co-d.c.e. sets. The first theorem shows that we can use the low co-d.c.e. enumeration degrees in place of $\mathcal{Z}$. Note that the class of co-d.c.e. enumeration degrees is the first class in the difference hierarchy that contains non-total elements. This is where we step outside the realm of the Turing degrees.

Theorem 9. Let $\mathbf{x}, \mathbf{y}$ be $\Delta_{2}^{0}$ enumeration degrees, such that $\mathbf{x}^{\prime}=\mathbf{0}_{e}^{\prime}$ and $\mathbf{y} \not \mathbf{x}$. There are $\Delta_{2}^{0}$ enumeration degrees $\mathbf{g}_{i}, \Pi_{1}^{0}$ enumeration degrees $\mathbf{a}_{i}$ and low co-d.c.e. enumeration degrees $\mathbf{c}_{i}, \mathbf{b}_{i}$ for $i=1,2$, such that:

(1) $\mathbf{g}_{i}$ is the least element below $\mathbf{a}_{i}$ which joins $\mathbf{b}_{i}$ above $\mathbf{c}_{i}$;

(2) $\mathbf{x} \leq \mathbf{g}_{1} \vee \mathbf{g}_{2}$

(3) $\mathbf{y} \not \leq \mathbf{g}_{1} \vee \mathbf{g}_{2}$.

The second theorem determines the position of every low co-d.c.e. enumeration degree among all $\Delta_{2}^{0}$ enumeration degrees using the $\Pi_{1}^{0}$ enumeration degrees. 
Theorem 10. Suppose $\mathbf{x}$ is a co-d.c.e. enumeration degree, $\mathbf{x}^{\prime}=\mathbf{0}_{e}^{\prime}$ and $\mathbf{y}$ is a $\Delta_{2}^{0}$ enumeration degree, such that $\mathbf{y} \not \mathbf{x}$. There are $\Delta_{2}^{0}$ enumeration degrees $\mathbf{g}_{i}$ and $\Pi_{1}^{0}$ enumeration degrees $\mathbf{a}_{i}, \mathbf{c}_{i}, \mathbf{b}_{i}$ for $i=1,2$, such that:

(1) $\mathbf{g}_{i}$ is the least element below $\mathbf{a}_{i}$ which joins $\mathbf{b}_{i}$ above $\mathbf{c}_{i}$;

(2) $\mathbf{x} \leq \mathbf{g}_{1} \vee \mathbf{g}_{2}$;

(3) $\mathbf{y} \not \leq \mathbf{g}_{1} \vee \mathbf{g}_{2}$.

The proofs of these two theorems are lengthy and technical. We will leave them for Section 7 and Section 8 respectively.

Before we proceed with the proof of the main result for this section we observe the following consequence of Theorem 8, Theorem 9 and Theorem 10. We say that a function $f$ fixes an element $a$ if $f(a)=a$.

Corollary 1. If $\pi: \mathcal{D}_{e}\left(\leq \mathbf{0}_{e}^{\prime}\right) \rightarrow \mathcal{D}_{e}\left(\leq \mathbf{0}_{e}^{\prime}\right)$ is an automorphism which fixes all $\Pi_{1}^{0}$ enumeration degrees, then $\pi$ fixes:

(1) all low co-d.c.e. enumeration degrees;

(2) all low $\Delta_{2}^{0}$ enumeration degrees;

(3) all total $\Delta_{2}^{0}$ enumeration degrees.

Proof. Let $\pi$ be an automorphism of $\mathcal{D}_{e}\left(\leq \mathbf{0}_{e}^{\prime}\right)$, which fixes all $\Pi_{1}^{0}$ enumeration degrees. Consider first a low co-d.c.e. enumeration degree $\mathbf{x}$ and let $\mathbf{y}=\pi(\mathbf{x})$. From the definability of the low enumeration degrees it follows that $\mathbf{y}$ is low, and hence $\Delta_{2}^{0}$. By Theorem 10 it must be that $\mathbf{y} \leq \mathbf{x}$. Now consider $\mathbf{z}=\pi^{-1}(\mathbf{x})$. This too must be a low enumeration degree. Noting that $\pi^{-1}$ is also an automorphism which fixes all $\Pi_{1}^{0}$ enumeration degrees we have in this case as well by Theorem 10 that $\mathbf{z} \leq \mathbf{x}$. So we have that $\mathbf{z} \leq \mathbf{x}$ and hence $\pi(\mathbf{z}) \leq \pi(\mathbf{x})$, i.e. $\mathbf{x} \leq \mathbf{y}$. But we already know $\mathbf{y} \leq \mathbf{x}$, thus $\mathbf{x}=\pi(\mathbf{x})$.

The other two statements follow in a similar way from Theorem 9 and Theorem 8 respectively, using the fact that the low and the total enumeration degrees are definable subclasses of the $\Delta_{2}^{0}$ enumeration degrees.

Proof of Theorem \%. Let $\vec{p}$ be basic indexing parameters, $\mathcal{M}$ be the standard model of arithmetic defined by $\vec{p}$ and $\varphi$ be the basic indexing defined by $\vec{p}$. Let $\left\{U_{e}\right\}_{e<\omega}$ be an enumeration of all low co-d.c.e. sets, defined in the following way: let $e=$ $\left\langle e_{1}, e_{2}\right\rangle$ then $U_{e}=\overline{W_{e_{1}} \backslash W_{e_{2}}}$ if $\overline{W_{e_{1}} \backslash W_{e_{2}}}$ is of low enumeration degree and $U_{e}=\emptyset$ otherwise. Using parameters $\vec{p}$ we first define an indexing $\theta$ of $\left\{U_{e}\right\}_{e<\omega}$.

Let $\mathbf{e}$ be an enumeration degree. The property $\mathbf{e} \in \mathbb{N}^{\mathcal{M}}$ is definable from $\vec{p}$, so we may assume that $\mathbf{e}=e^{\mathcal{M}}$, for some natural number $e$. Recall that we have an effective way to translate an arithmetic statement about $e$, say $\psi(e)$, into a degree theoretic statement $\hat{\psi}(\mathbf{e}, \overrightarrow{\mathbf{p}})$ so that:

$$
\mathbb{N} \models \psi(e) \text { if and only if } \mathcal{D}_{e}\left(\leq \mathbf{0}_{e}^{\prime}\right) \models \hat{\psi}(\mathbf{e}, \overrightarrow{\mathbf{p}}) .
$$

We will say that $\psi(e)$ is true in $\mathcal{M}$ to mean that $\psi(\mathbf{e}, \overrightarrow{\mathbf{p}})$ is true in $\mathcal{D}_{e}\left(\leq \mathbf{0}_{e}^{\prime}\right)$. Using Theorem 10 and the definability of the low enumeration degrees we define $\theta(\mathbf{e})$ as the largest low enumeration degree in the set of all $\mathbf{x}$ with the following property: for every $\mathbf{a}_{i}, \mathbf{b}_{i}, \mathbf{c}_{i}$ and $\mathbf{g}_{i}$ where $i=1,2$ for which there are elements $e_{a_{i}}^{\mathcal{M}}, e_{b_{i}}^{\mathcal{M}}$ and $e_{c_{i}}^{\mathcal{M}}$, such that $\varphi\left(e_{a_{i}}^{\mathcal{M}}\right)=\mathbf{a}_{i}, \varphi\left(e_{b_{i}}^{\mathcal{M}}\right)=\mathbf{b}_{i}$ and $\varphi\left(e_{c_{i}}^{\mathcal{M}}\right)=\mathbf{c}_{i}$, and $\mathbf{g}_{i}$ is the least element below $\mathbf{a}_{i}$ which joins $\mathbf{b}_{i}$ above $\mathbf{c}_{i}$ for $i=1,2$ and in $\mathcal{M}$ the statement:

" $d_{e}\left(U_{e}\right)$ is bounded by the join of the least enumeration degree below $d_{e}\left(\overline{W_{e_{a_{1}}}}\right)$ which joins $d_{e}\left(\overline{W_{e_{b_{1}}}}\right)$ above $d_{e}\left(\overline{W_{e_{c_{1}}}}\right)$; and the least 
enumeration degree below $d_{e}\left(\overline{W_{e_{a_{2}}}}\right)$ which joins $d_{e}\left(\overline{W_{e_{b_{2}}}}\right)$ above $d_{e}\left(\overline{W_{e_{c_{2}}}}\right) . "$

is true, then $\mathbf{x} \leq \mathbf{g}_{1} \vee \mathbf{g}_{2}$.

Next we use Theorem 9 to define an indexing $\psi$ of the sequence $V_{e}$, where $V_{e}=W_{e}\left(K_{\emptyset} \oplus \overline{K_{\emptyset}}\right)$, if this is a low set, and $V_{e}=\emptyset$ otherwise. Assume that $\mathbf{e}=e^{\mathcal{M}}$. Then $\psi(\mathbf{e})$ is the largest enumeration degree out of all low enumeration degrees $\mathbf{x}$ with the following property: for every $\mathbf{a}_{i}, \mathbf{b}_{i}, \mathbf{c}_{i}$ and $\mathbf{g}_{i}$ where $i=1,2$ for which there are elements $e_{a_{i}}^{\mathcal{M}}, e_{b_{i}}^{\mathcal{M}}$ and $e_{c_{i}}^{\mathcal{M}}$, such that $\varphi\left(e_{a_{i}}^{\mathcal{M}}\right)=\mathbf{a}_{i}, \theta\left(e_{b_{i}}^{\mathcal{M}}\right)=\mathbf{b}_{i}$ and $\theta\left(e_{c_{i}}^{\mathcal{M}}\right)=\mathbf{c}_{i}$, and $\mathbf{g}_{i}$ is the least element below $\mathbf{a}_{i}$ which joins $\mathbf{b}_{i}$ above $\mathbf{c}_{i}$ for $i=1,2$ and in $\mathcal{M}$ the statement:

" $d_{e}\left(V_{e}\right)$ is bounded by the join of: the least e-degree below $d_{e}\left(\overline{W_{e_{a_{1}}}}\right)$ which joins $d_{e}\left(U_{e_{b_{1}}}\right)$ above $d_{e}\left(U_{e_{c_{1}}}\right)$; and the least e-degree below $d_{e}\left(\overline{W_{e_{a_{2}}}}\right)$ which joins $d_{e}\left(U_{e_{b_{2}}}\right)$ above $d_{e}\left(U_{e_{c_{2}}}\right)$."

is true then $\mathbf{x} \leq \mathbf{g}_{1} \vee \mathbf{g}_{2}$.

Finally, we define an indexing $\delta$ of the sequence $\left\{X_{e} \oplus \overline{X_{e}}\right\}_{e<\omega}$, i.e. an indexing of the total $\Delta_{2}^{0}$ sets. Recall, that $X_{e}$ is the set with characteristic function $\{e\}^{\emptyset^{\prime}}$, if this is a total $\{0,1\}$-valued function and $\emptyset$ otherwise. Let $\mathbf{e}=e^{\mathcal{M}}$ for some natural number $e$. We let $\delta(\mathbf{e})=\psi\left(i^{\mathcal{M}}\right) \vee \psi\left(j^{\mathcal{M}}\right)$, where $i^{\mathcal{M}}$ and $j^{\mathcal{M}}$ are enumeration degrees satisfying the following arithmetic statement in $\mathcal{M}$ :

"The pair $(i, j)$ is lexicographically the least pair of indices, such that $V_{i} \oplus V_{j} \equiv_{e} X_{e} \oplus \overline{X_{e}}$ ".

That such a pair exists follows from Theorem 8 .

\section{Step 2: An indexing of the total $\mathcal{I} \mathcal{T}\left(\mathbf{0}_{e}^{\prime}\right)$ sets}

Recall that $\mathcal{I} \mathcal{T}\left(\mathbf{0}_{e}^{\prime}\right)$ consists of all intervals $\left[\mathbf{x}, \mathbf{x}^{\prime}\right]$, where $\mathbf{x} \leq \mathbf{0}_{e}^{\prime}$ is a total enumeration degree. The main goal of this section is to prove the following theorem:

Theorem 11. Let $\vec{p}$ be basic indexing parameters. Then $\vec{p}$ define:

(1) a $\mathbf{0}_{e}^{\prime}$-basic indexing.

(2) an indexing of the total $\mathcal{I} \mathcal{T}\left(\mathbf{0}_{e}^{\prime}\right)$ sets.

The second statement of this theorem will follow easily from the first statement using Theorem 8, Theorem 9 and Theorem 10 relativized above any total enumeration degree. The challenge is to prove the first statement. We need to show that we can determine the image of the Turing degrees (under the standard embedding $\iota$ ) of sets that are c.e. in and above some $\Delta_{2}^{0}$ set. Fix a total $\mathbf{b} \leq \mathbf{0}_{e}^{\prime}$ and let $\mathbf{a}$ be the image of a Turing degree that is c.e. in and above $\iota^{-1}(\mathbf{b})$. If $\mathbf{a} \geq_{e} \mathbf{0}_{e}$ then we can use Shoenfield's jump inversion theorem, the definability of the enumeration jump and the indexing of the total $\Delta_{2}^{0}$ sets to determine $\mathbf{a}$.

Theorem 12 (Shoenfield [14]). If $\mathbf{a}$ is c.e. in and above $\mathbf{0}^{\prime}$ then $\mathbf{a}=\mathbf{x}^{\prime}$ for some total $\mathbf{x} \leq \mathbf{0}^{\prime}$

So let us assume that $\mathbf{a}$ is not above $\mathbf{0}_{e}^{\prime}$. We can reduce our task further by using a relativized form of Sack's splitting theorem with cone avoidance:

Theorem 13 (Sacks [12]). Let $\mathbf{x}$ and $\mathbf{y}$ be nonzero c.e. Turing degrees, such that $\mathbf{y}$ is nonzero. There are low c.e. Turing degrees $\mathbf{a}$ and $\mathbf{b}$, such that $\mathbf{x}=\mathbf{a} \vee \mathbf{b}$ and $\mathbf{a} \ngtr \mathbf{y}$ and $\mathbf{b} ¥ \mathbf{y}$. 
What is left is to determine $\mathbf{a}$, assuming it is low relative to $\mathbf{b}$ and not above $\mathbf{0}_{e}^{\prime}$. The definability of the jump operation and the image of the relation "c.e. in" will let us pick out the images of all degrees $\mathbf{y}$ which are low relative to $\mathbf{b}$, not above $\mathbf{0}^{\prime}$ and c.e. in $\mathbf{b}$. The following theorem relativized to $\mathbf{b}$ shows that $\mathbf{a}$ can be distinguished among all images of such $\mathbf{y}$ using only elements below $\mathbf{0}_{e}^{\prime}$.

Theorem 14. If $A$ and $W$ are c.e. sets and $Y$ is a low c.e. set then one of the following is true:

(1) $W \leq_{T} Y$;

(2) $A \leq_{T} Y$;

(3) There are sets $U$ and $V$ computable from $W$, such that $A$ joins $U$ above $V$, whereas $Y$ does not join $U$ above $V$.

Proof. Let $A$ and $W$ be c.e. sets and let $Y$ be a low c.e. set. Suppose that $W \not_{T} Y$ and $A \not_{T} Y$. We will build $\Delta_{2}^{0}$ approximations $\{U[s]\}_{s<\omega}$ and $\{V[s]\}_{s<\omega}$ to sets $U$ and $V$. We will only enumerate $n$ in $U$ or $V$ if a number less than $n$ enters $W$ at the same stage. This ensures that $U$ and $V$ are computable from $W$.

To ensure that $A \oplus U \geq_{T} V$ we use a movable marker strategy. To every natural number $x$ we will attach a marker $\gamma(x)$, initially $\gamma(x)=x+1$. The value of the marker can be raised during the construction if we observe a change in $A \uparrow \gamma(x)$ or we make a change in $U \uparrow \gamma(x)$. Similarly, we will only change the value of $V(x)$ provided that we can raise the value of $\gamma(x)$. The construction will ensure that every marker reaches a finite limit value. Then $V(x)=V[s](x)$, where $s$ is the least stage in the construction at which $A[s]$ and $U[s]$ are correct up to $\gamma(x)[s]$.

Let $\left\{\Theta_{e}\right\}_{e<\omega}$ be a listing of all Turing functionals. For every $e$ we have to also satisfy the requirement:

$$
\mathcal{R}_{e}: \Theta_{e}^{Y \oplus U} \neq V
$$

The strategy for $\mathcal{R}_{e}$ is to search for an opportunity to diagonalize $\Theta_{e}^{Y \oplus U}$ against $V$. If at stage $s$ we find a number $x$, such that $\Theta_{e}^{Y \oplus U}(x)$ is defined at stage $s$ and the computation has use $\theta_{e}(x)<\gamma(x)$, then we will call $x$ an opportunity for $e$ at stage $s$. The opportunity is realized once a number $y<x$ enters $W$. We can then change $U(\gamma(x))$, while preserving $U\left\lceil\theta_{e}(x)\right.$, and set $V(x) \neq \Theta_{e}^{Y \oplus U}$.

If $Y[s] \uparrow \theta_{e}(x)=Y \uparrow \theta_{e}(x)$ then we say that $x$ is a correct opportunity. In this case the diagonalization will remain true at all further stages. If $Y$ is incorrect infinitely often, we risk making $\gamma(x)$ unbounded. We use the fact that $Y$ is low and hence we have $\Delta_{2}^{0}$ approximations to sets computable from $Y^{\prime}$, to avoid this. We approximate whether or not $x$ is a correct opportunity as follows. Using the recursion theorem we can assume we know an index of this construction and so we can find a $\Delta_{2}^{0}$ approximation to the set

$$
Q_{e}(w)=\left\{x \mid \exists t>w\left(\theta_{e}(x)[t]<\gamma(x)[t] \&\left(Y \uparrow \theta_{e}(x)\right)[t] \preceq Y\right)\right\} .
$$

Here $w$ is meant to be the first stage since $\mathcal{R}_{e}$ started working with $x$, recorded as $w_{e}(x)$ in the construction. Once $\alpha$ starts working with $x$ at stage $w_{e}(x)$ at every further stage it checks if $x \in Q_{e}\left(w_{e}(x)\right)$. While this is true, the strategy tries to turn $x$ into an opportunity by using every possibility to raise the value of $\gamma(x)$ above $\theta_{e}(x)$. Changes in $A$ give us such possibilities.

If $x \notin Q_{e}\left(w_{e}(x)\right)$, but $\Theta_{e}^{Y, U}(x) \downarrow$ then there is an inconsistency. We can speed up the approximations to $\Theta_{e}, Y$ and $Q_{e}$, until we either observe that the computation 
was incorrect or $x$ enters $Q_{e}\left(w_{e}(x)\right)$. If $x \notin Q_{e}\left(w_{e}(x)\right)$ permanently then the strategy will give up on $x$ as a diagonalization witness.

This process will be repeated in turn for all $x>e$ until a permanent diagonalization is achieved. If there are infinitely many $Y$-correct opportunities, none of which are allowed to be realized by $W$ then we will argue that $W \leq_{T} Y$. If there are only finitely many $Y$-correct opportunities for $e$ and $\Theta_{e}^{Y \oplus U}$ is total then we will show that $A \leq_{T} Y$.

Before we proceed to describe a formal construction, we need to consider how all of the strategies work together. This complicates matters as now $U$ can change due to actions by other strategies. On the other hand if $\Theta_{e}^{Y, U}$ is not total, there is a risk that infinite activity of one strategy interferes with the work of lower

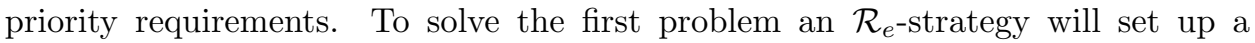
restraint $R(e+1)$, and require that lower priority strategies do not modify $U$ or $V$ on numbers less than $R(e+1)$. To solve the second problem, we use that $Y$ is low once again to approximate the set

$$
T_{e}(w)=\left\{x \mid \exists s>w\left(\Theta_{e}^{Y, U}(x)[s] \downarrow \& Y[s] \uparrow \theta_{e}(x)=Y \uparrow \theta_{e}(x)\right)\right\} .
$$

The $\mathcal{R}_{e}$ strategy will act only for numbers $x \in T_{e}\left(w_{e}(x)\right)$. We will assume that Turing functionals are defined so that if $y<x$ and $\Theta^{Z}(x)[s] \downarrow$ then $\Theta^{Z}(y)[s] \downarrow$ and $\theta(y)<\theta(x)$. Thus, if $\Theta_{e}^{Y, U}(x) \uparrow$ for some number $x$ then eventually $\mathcal{R}_{e}$ will stop acting.

\section{Construction}

At stage $0, U_{0}=V_{0}=\emptyset, \gamma(x)[0]=x+1$ for all $x$, the restraint $R(e)[0]=0$ for every $e$, the stage $w_{e}(x)$ at which a number $x$ starts being examined by $\mathcal{R}_{e}$ is initially undefined. At stage $s>0$ we examine the requirements $\mathcal{R}_{0} \ldots \mathcal{R}_{s-1}$ in turn until one of them terminates the stage. Unless otherwise specified all parameters inherit their values from the previous stage. If a requirement $\mathcal{R}_{e}$ is cancelled at stage $s$ then $\mathrm{d} w_{e}(x)$ is set to $s+1$ for all $x$.

Requirement $\mathcal{R}_{e}$ : Examine each $x$, such that $R(e)<x<s$, in turn until an instruction forces us stop.

First we ensure that the approximation to $Y$ and to the sets $T_{e}$ and $Q_{e}$ agree about $x$. We will say that they agree if

- $\Theta_{e}^{Y, U}(x)[s] \uparrow$ and $x \notin T_{e}\left(w_{e}(x)\right)[s]$ or

- $\Theta_{e}^{Y, U}(x)[s] \downarrow, x \in T_{e}\left(w_{e}(x)\right)[s]$ and $x \in Q_{e}\left(w_{e}(x)\right)[s]$.

- $\Theta_{e}^{Y, U}(x)[s] \downarrow, x \in T_{e}\left(w_{e}(x)\right)[s], x \notin Q_{e}\left(w_{e}(x)\right)[s]$ and $\theta_{e}(x)[s] \geq \gamma(x)[s]$.

If none of the cases above hold then we speed up the approximations to $Y, \Theta_{e}$, $T_{e}\left(w_{e}\right)$ and $Q_{e}\left(w_{e}\right)$ until they do. We pick the first case which applies to $x$.

(1) If $x \notin T_{e}\left(w_{e}(x)\right)[s]$ then we cancel all $w_{e}(y)$ for $y>x$ and move on to the next requirement.

(2) If $R(e+1) \leq \theta_{e}(x)[s]$ or $R(e+1)<R(e)$ then we redefine $R(e+1)$ as $\max \left(R(e), \theta_{e}(x)[s]+1\right)$. We cancel all lower priority requirements and all $w_{e}(y)$ for $y>x$ and end this stage.

(3) If $\theta_{e}(x)[s] \geq \gamma(x)$ and $A(z)[s-1] \neq A(z)[s]$ for some $z<\gamma(x)$ then we redefine $\gamma(y)$ for all $y \geq x$ to be numbers larger than any seen in the construction so far. We cancel all lower priority requirements and all $w_{e}(y)$ for $y>x$ and end this stage.

(4) If $x \notin Q_{e}\left(w_{e}(x)\right)[s]$ then we move on to $x+1$. 
(5) If $V(x)[s] \neq \Theta_{e}^{Y, U}(x)[s]$ then we cancel all $w_{e}(y)$ for $y>x$ and move on to the next requirement.

(6) If $\theta_{e}(x)[s]<\gamma(x)$ and $W(z)[s-1] \neq W(z)[s]$ for some $z<x$ then we set $V(x)[s]=1-\Theta_{e}^{Y, U}(x)[s]$. Set $U(\gamma(x))[s]=1$ and let reset $\gamma(y)$ for all $y \geq x$ to be fresh numbers. Finally we set $R(e+1)=\gamma(x)+1$. Cancel all lower priority requirements and all $w_{e}(y)$ for $y>x$ and end this stage.

(7) If none of the above cases applies to $x$ then move on to $x+1$.

If all $x$ are scanned without ending the stage then move on to the next requirement. End of Construction

We will show by induction on $e$ that $\mathcal{R}_{e}$ is satisfied. We will furthermore show that there is a stage $s_{e+1}$, such that at every $t>s_{e+1}$ the $\mathcal{R}_{e}$ strategy does not initialize lower priority strategies or change the value of any parameter. Assume inductively that this is true for $i<e$. We can find a stage $s_{e}$, such that at all stages $t>s_{e}, \mathcal{R}_{e}$ is examined, not initialized, the value of $R(e)$ is fixed and the $\gamma$-markers for $x \leq R(e)$ do not change.

Fix $x>R(e)$ and assume that after stage $s_{x} \geq s_{e}$, the strategy $\mathcal{R}_{e}$ does not change any parameters when examining $y<x$. Then after stage $s_{x}$ the value of $w_{e}(x)$ does not change and $x$ is examined by $\mathcal{R}_{e}$ at every stage $t>s_{x}$. If $x \notin T_{e}\left(w_{e}(x)\right)$ then $\Theta_{e}^{Y, U}$ is undefined at $x$. Let $s_{e+1}$ be the stage at which the approximation to $T_{e}\left(w_{e}(x)\right)$ stabilizes on $x$. Then at every stage $t>s_{e+1}$ the strategy $\mathcal{R}_{e}$ moves on to the next requirement under Case (1) for $x$, without further actions.

If $x \in T_{e}\left(w_{e}(x)\right)$ then as $x$ is examined at every stage $t$ after the last time $w_{e}(x)$ was changed, let $s_{1}>w_{e}(x)$ be the least stage, such that $\Theta_{e}^{Y, U}(x)\left[s_{1}\right]$ is defined and $Y$-correct. Then after stage $s_{1}$ Case(1) will not apply for $x$ anymore. We pass through Case 2 at stage $s_{1}$ if $R(e+1)\left[s_{1}\right] \leq \theta_{e}(x)\left[s_{1}\right]$. Hence the $Y$-correct computation is preserved at all stages $t>s_{1}$ and Case (1) or (2) will never again apply for $x$. As $\theta_{e}(x)$ does not change after stage $s_{1}$ it follows that there can be at most one stage $t>s_{1}$, such that Case (3) applies to $x$. If there is such a stage then we move the value of $\gamma(x)$ above $\theta_{e}(x)$ permanently.

Let $s_{2} \geq s_{1}$ be the least stage such the approximation $Q_{e}\left(w_{e}(x)\right)$ stabilizes on $x$ at stage $s_{2}$ and Cases (1) (2) and (3) do not apply for $x$. If $x \notin Q_{e}\left(w_{e}(x)\right)$ then at every stage $t>s_{2}$ Case (4) applies for $x$, the strategy does not change any parameters when examining $x$ and $x+1$ is always accessible. We say that $x$ is not an opportunity.

Suppose that $x \in Q_{e}\left(w_{e}(x)\right)$. Then there is a stage $t \geq w_{e}(x)$, such that $\theta_{e}(x)[t]<\gamma(x)[t]$ and $Y[t]\left\lceil\theta_{e}(x) \preceq Y\right.$. As $s_{1}$ is the least stage at which the computation $\Theta^{Y, U}$ is $Y$-correct and we preserved it at all further stages, it follows that $\theta_{e}(x)<\gamma(x)[t]$ at all $t>s_{2}$. If there is a stage $s_{3} \geq s_{2}$ such $V(x)\left[s_{3}\right] \neq \Theta_{e}^{Y, U}(x)\left[s_{3}\right]$ then this inequality is preserved at all further stages thus $\mathcal{R}_{e}$ is permanently. In particular if at any stage $t \geq s_{2}$ Case (6) applies then at stage $t+1$ Case 5 will apply for $x$. At every stage $t>s_{3} \mathcal{R}_{e}$ moves on to the next requirement under Case (5) for $x$. It does not change $R(e+1)$ or change any $\Gamma$-marker.

The only other option for $x$ is that at every stage $t>s_{2}$ Case(7) applies for $x$. In this case as well the $\Gamma$-markers for all $y \leq x$ do not change after stage $s_{2}$ and $\mathrm{x}$ does not cancel the values of $w_{e}(y)$ for $y>x$. We will say that $x$ is an unrealized opportunity. 
It follows from the analysis above that for every $x$ the value of $\gamma(x)$ reaches a limit and so $V \leq_{T} A \oplus U$.

If $\Theta_{e}^{U, Y}$ is not total then eventually $\mathcal{R}_{e}$ will reach a number $x$, such that $x \notin$ $T_{e}\left(w_{e}(x)\right)$ and so $\mathcal{R}_{e}$ will always move onto $\mathcal{R}_{e+1}$ under Case (1)when examining $x$, without further actions. Suppose that $\Theta_{e}^{U, Y}$ is total. We claim that there is a largest $x$, examined by $\mathcal{R}_{e}$ at all but finitely many stages, such that $x$ is a realized correct opportunity and $\Theta_{e}^{U, Y}(x) \neq V(x)$. In this case as well $\mathcal{R}_{e}$ will always move onto $\mathcal{R}_{e+1}$ under Case (5) without further action, thus completing the induction step.

Assume towards a contradiction that every $x>R(e)$ is either not an opportunity or an unrealized opportunity. Suppose that there is a number $x_{0}$, such that every $x>$ $x_{0}$ is not an opportunity. We show that $A \leq_{T} Y$, contradicting our assumptions. Fix a number $x>x_{0}$. To compute $A(x)$ we find a stage $s$ in the contraction, such that $\Theta_{e}^{U, Y}(x)[s] \downarrow$ and the computation is $Y$-correct. Then $A(x)=A(x)[s]$. Indeed if $A(x)$ changes after stage $s$ then we will move the marker $\gamma(x)$ above $\theta(x)$ and $x$ will become an opportunity.

If there are infinitely many unrealized opportunities then we show that $W \leq_{T} Y$, contradicting our assumptions: to compute $W(z)$, search for an element $x>z$ and a stage $s$ such that $\Theta_{e}^{Y, U}(x)[s] \downarrow$ and is $Y$-correct and $\theta_{e}(x)<\gamma(x)$. As there are infinitely many unrealized opportunities we will eventually find such a pair of elements $s$ and $x$. Then $W(z)=W(z)[s]$, otherwise $x$ would become realized.

Proof of Theorem 11. Let $\vec{p}$ be basic indexing parameters and let $\mathcal{M}$ and $\delta$ be the definable from $\vec{p}$ model of arithmetic and indexing of the total $\Delta_{2}^{0}$ sets. We will use $\vec{p}$ to define a $\mathbf{0}_{e}^{\prime}$-basic indexing $\varphi^{\mathbf{0}_{e}^{\prime}}$, i.e. an indexing of the sequence $\left\{Y_{e} \oplus \overline{Y_{e}}\right\}_{e<\omega}$, where $Y_{e}=X_{e_{1}} \oplus W_{e_{0}}^{X_{e_{1}}}$ and $X_{e_{1}}$ is the $e_{1}$-th $\Delta_{2}^{0}$ set, whenever $e=\left\langle e_{0}, e_{1}\right\rangle$. So fix $\mathbf{e}$ and let $e$ be a natural number, such that $e^{\mathcal{M}}=\mathbf{e}$. We determine $\varphi^{\mathbf{0}_{e}^{\prime}}(\mathbf{e})$ according to the following cases:

Case 1: If in $\mathcal{M}$ we have that $\emptyset^{\prime} \leq_{T} Y_{e}$, then we use Theorem 12 to find $i^{\mathcal{M}}$, such that in $\mathcal{M}$ the following statement is true:

" $X_{i}^{\prime}$ is Turing equivalent to $Y_{e} . "$

We then set $\varphi^{\mathbf{0}_{e}^{\prime}}(\mathbf{e})=\delta\left(i^{\mathcal{M}}\right)^{\prime}$ using that the enumeration jump is definable by Theorem 1 and that the standard embedding $\iota$ preserves the jump operation.

Case 2: If in $\mathcal{M}$ we have that $Y_{e}^{\prime} \equiv_{T} X_{e_{1}}^{\prime}$ and $\emptyset^{\prime} \not_{T} Y_{e}$ then by Theorem 14 relativized to $X_{e_{1}}$ we have that if $Y$ is c.e. in and above $X_{e_{1}}$ and does not compute $\emptyset^{\prime}$ or $Y_{e}$ then there are $\Delta_{2}^{0}$ sets $U$ and $V$, such that $Y_{e}$ joins $U$ above $V$, whereas $Y$ does not join $U$ above $V$. Using that the image of the relation "c.e. in" is definable by Theorem 5 we can determine $\varphi^{\emptyset^{\prime}}(\mathbf{e})$ as the least element in the set of all $\mathbf{x} \geq \delta\left(e_{1}^{\mathcal{M}}\right)$ that satisfy the following statement: $\mathbf{x}^{\prime}=\delta\left(e_{1}^{\mathcal{M}}\right)^{\prime}, \mathbf{0}_{e}^{\prime} \not \mathbf{x}, \iota^{-1}(\mathbf{x})$ is c.e. in $\iota^{-1}\left(\delta\left(e_{1}^{\mathcal{M}}\right)\right)$ and $\delta\left(j^{\mathcal{M}}\right) \leq \mathbf{x} \vee \delta\left(i^{\mathcal{M}}\right)$ for every $i$ and $j$, such that in $\mathcal{M}$ the following statement is true

" $Y_{e}$ joins $X_{i}$ above $X_{j} . "$

Case 3: If none of the case above are true then we use Theorem 13 to find $i$ and $j$, such that in $\mathcal{M}$ :

" $Y_{i}$ and $Y_{j}$ are c.e. in and above $X_{e_{1}}$, they are low over $X_{e_{1}}$, they do not compute $\emptyset^{\prime}$ and $Y_{e} \equiv_{T} Y_{i} \oplus Y_{j}$." 
We use Case 2 to define $\varphi^{\mathbf{0}_{e}^{\prime}}\left(i^{\mathcal{M}}\right)$ and $\varphi^{\mathbf{0}_{e}^{\prime}}\left(i^{\mathcal{M}}\right)$ and $\operatorname{set} \varphi^{\mathbf{0}_{e}^{\prime}}(\mathbf{e})=\varphi^{\mathbf{0}_{e}^{\prime}}\left(i^{\mathcal{M}}\right) \vee$ $\varphi^{\mathbf{0}_{e}^{\prime}}\left(j^{\mathcal{M}}\right)$.

Now that we have defined $\varphi^{\mathbf{0}_{e}^{\prime}}$ we use it together with Theorem 8, Theorem 9 and Theorem 10 relativized above any total enumeration degree to define an indexing $\xi^{\mathbf{0}_{e}^{\prime}}$ of the total sets in $\mathcal{I T}\left(\mathbf{0}_{e}^{\prime}\right)$, i.e. of the sequence $\left\{Z_{e} \oplus \overline{Z_{e}}\right\}_{e<\omega}$, where $e=\left\langle e_{0}, e_{1}\right\rangle$ and $Z_{e}=\left\{e_{0}\right\}^{X_{e_{1}}}$ if this is a total $\{0,1\}$ valued function and $Z_{e}=\emptyset$ otherwise. The proof follows the definition of $\delta$ from Theorem 7 .

\section{Step 3: An indexing of the total $\Delta_{3}^{0}$ Sets}

The final step in our proof gives a method to obtain an indexing of the total $\Delta_{3}^{0}$ set form an indexing of the total sets in $\mathcal{I} \mathcal{T}\left(\mathbf{0}_{e}^{\prime}\right)$. Let $\mathbf{x}$ be a Turing degree below $\mathbf{0}^{\prime \prime}$. If $\mathbf{x} \geq \mathbf{0}^{\prime}$ we can use Theorem 11 to determine the image of $\mathbf{x}$ under the standard embedding. So suppose that $\mathbf{x} \nsucceq \mathbf{0}^{\prime}$. We will use genericity to determine $\mathbf{x}$ in three steps. Consult [19] for basic properties of generic sets.

Proposition 1. Let $n$ be a natural number and $\mathbf{x}$ be a Turing degree. If $\mathbf{x} \leq \mathbf{0}^{(n+1)}$ then $\mathbf{x}=\left(\mathbf{y}_{1} \vee \mathbf{y}_{2}\right) \wedge\left(\mathbf{y}_{3} \vee \mathbf{y}_{4}\right)$, where for each $i=1,2,3,4$ we have that $\mathbf{y}_{i}^{\prime} \leq \mathbf{0}^{(n+1)}$.

Proof. If $\mathbf{x}=\mathbf{0}^{(n+1)}$ then we use Sacks' splitting theorem (Theorem 13) relativized to $\mathbf{0}^{(n)}$ to split $\mathbf{x}$ into two low relative to $\mathbf{0}^{(n)}$ degrees $\mathbf{y}_{1}$ and $\mathbf{y}_{2}$. Then $\mathbf{x}=$ $\left(\mathbf{y}_{1} \vee \mathbf{y}_{2}\right) \wedge\left(\mathbf{y}_{1} \vee \mathbf{y}_{2}\right)$. So suppose $\mathbf{x}<\mathbf{0}^{(n+1)}$ and let $i+1$ be the least number, such that $\mathbf{x} \geq \mathbf{0}^{(i+1)}$.

Fix $X \in \mathbf{x}$. Recall that a set $Y$ is 1-generic relative to $X$ if $Y$ meets or avoids every $\Sigma_{1}^{0}(X)$ set of finite binary strings. Every incomputable c.e. set computes a 1-generic set. Hence relativizing $X \oplus \emptyset^{(i+1)}$ computes a 1-generic relative to $X$ set $Y$. We split $Y$ into odd and evens numbers $Y=U \oplus V$. The sets $U$ and $V$ are mutually 1-generic relative to $X$, hence $U \oplus X$ and $V \oplus X$ form a minimal pair relative to $X$. We further transform $U \oplus X$ and $V \oplus X$ using the functional $C$.

The functional $C$ maps a pair of sets $X$ and $G$ to a set $C(X, G)$ as follows. Let $G=G_{\text {even }} \oplus G_{\text {odd }}$ :

$C(X, G)(n)= \begin{cases}G_{\text {even }}(n-m) & \text { if } G_{\text {odd }}(n)=0 \text { and } \mid G_{\text {odd }}\lceil n \mid=m \\ X(m) & \text { if } G_{\text {odd }}(n)=1 \text { and } n \text { is the } m \text {-th element of } G_{\text {odd }} .\end{cases}$

Note that $G \oplus X \equiv_{T} G_{\text {odd }} \oplus C(X, G)$. Let $\mathbf{y}_{1}=d_{T}\left(U_{\text {odd }}\right), \mathbf{y}_{2}=d_{T}(C(X, U))$, $\mathbf{y}_{3}=d_{T}\left(V_{\text {odd }}\right)$ and $\mathbf{y}_{4}=d_{T}(C(X, V))$. Then $\mathbf{x}=\left(\mathbf{y}_{1} \vee \mathbf{y}_{2}\right) \wedge\left(\mathbf{y}_{3} \vee \mathbf{y}_{4}\right)$.

In [16] we showed that if $G$ is 1-generic relative to $X$ then so is $C(X, G)$. If $G$ is 1 -generic then $G^{\prime} \leq_{T} G \oplus \emptyset^{\prime}$. Hence for every $i=1,2,3,4$ we have that $\mathbf{y}_{i}^{\prime} \leq \mathbf{0}^{(n+1)}$.

We have reduced our task to finding a way to determine only the total degrees $\mathbf{x}$ with $\mathbf{x}^{\prime} \leq \mathbf{0}_{\mathbf{e}}^{\prime \prime}$. We will reduce it further to determining only the images of the 2 -generic degrees. Recall, that a set is $(n+1)$-generic, if it is generic relative to $\emptyset^{(n)}$ and a degree is $(n+1)$-generic, if it contains an $(n+1)$-generic set.

Proposition 2. If $\mathbf{x}^{\prime} \leq \mathbf{0}^{(n+1)}$ then there are $(n+1)$-generic degrees $\mathbf{g}_{i} \leq \mathbf{0}^{(n+1)}$, $i=1,2,3,4$, such that $\mathbf{x}=\left(\mathbf{g}_{1} \vee \mathbf{g}_{2}\right) \wedge\left(\mathbf{g}_{3} \vee \mathbf{g}_{4}\right)$.

Proof. Fix $X \in \mathbf{x}$. As in the proof of Proposition 1 if $G$ is 1 -generic relative to $X$, then we can split it into even and odd bits $L \oplus R$, and then use the functional $C$ to define $\mathbf{g}_{1}=d_{T}\left(L_{o d d}\right), \mathbf{g}_{2}=d_{T}(C(X, L)), \mathbf{g}_{3}=d_{T}\left(R_{o d d}\right)$ and $\mathbf{g}_{4}=d_{T}(C(X, R))$. 
We would like to ensure that all of the elements in this definition of $\mathbf{x}$ are also $(n+1)$-generic. It will be sufficient to ensure that $G, C(X, L)$ and $C(X, R)$ are $(n+1)$-generic.

We extend the definition of $C$ so that it can have a finite binary string of even length as a second parameter. Given a finite binary string $\tau$, we can break it up into two finite binary strings $\tau_{\text {even }}$ and $\tau_{\text {odd }}$, so that $\tau_{\text {even }}(n)=\tau(2 n)$ and $\tau_{\text {odd }}(n)=\tau(2 n+1)$. We will write $\tau=\tau_{\text {even }} \oplus \tau_{\text {odd }}$.

$$
C(Y, \tau)(n)= \begin{cases}\tau_{\text {even }}(n-m) & \text { if } \tau_{\text {odd }}(n)=0 \text { and }\left|\left\{x<n \mid \tau_{\text {odd }}(x)=1\right\}\right|=m ; \\ Y(m) & \text { if } \tau_{\text {odd }}(n)=1 \text { and } n \text { is the } m \text {-th element of } \tau_{\text {odd }} \\ \uparrow & \text { if } \tau_{\text {odd }}(n) \uparrow .\end{cases}
$$

For every $Z$ let $D_{e}(Z)$ denote the dense set $D_{e}(Z)=\left\{\sigma \mid \sigma \in W_{e}^{Z}\right\} \cup\{\sigma \mid \forall \tau \succeq$ $\left.\sigma\left(\tau \notin W_{e}^{Z}\right)\right\}$. Note that for every $e, D_{e}(Z)$ is computable from $Z^{\prime}$. We need to ensure that $G$ meets $D_{e}(X)$ and that $G, C(X, L)$ and $C(X, R)$ meet $D_{e}\left(\emptyset^{(n)}\right)$ for every $e$. As $X^{\prime} \leq_{T} \emptyset^{(n+1)}$ it follows that these sets are computable from $\emptyset^{(n+1)}$. We build $G$ as $\bigcup_{s} \sigma[s]$ by the finite extension method using oracle $\emptyset^{(n+1)}$. Let $\sigma_{0}=\emptyset$. Suppose we have constructed $\sigma[s]$.

(1) If $s=\langle 0, e\rangle$ then $\sigma[s+1]$ is the least extension of $\sigma[s]$ in $D_{e}(X)$.

(2) If $s=\langle 1, e\rangle$ then $\sigma[s+1]$ is the least extension of $\sigma[s]$ in $D_{e}\left(\emptyset^{(n)}\right)$.

(3) If $s=\langle 2, e\rangle$ then let $\sigma[s]=\sigma_{L} \oplus \sigma_{R}$. We can assume that $\left|\sigma_{L}\right|$ is even. Let $\rho=C\left(X, \sigma_{L}\right)$. We would like to extend $\sigma[s]$ so that $\rho$ has an extension in $D_{e}\left(\emptyset^{(n)}\right)$. Find a finite binary string $\tau$, such that $\hat{\rho^{\wedge} \tau}$ in $D_{e}\left(\emptyset^{(n)}\right)$. Let $k=|\tau|$. We can represent $\hat{\rho} \tau$ as $C\left(X, \sigma_{L} \hat{\tau^{*}}\right)$ where $\tau^{*}$ is of length $2 k$ and is defined as $\tau(0)^{\wedge} 0^{\wedge} \tau(1) \ldots{ }^{\wedge} 0^{\wedge} \tau(k)^{\wedge} 0$, i.e. the even bits correspond to $\tau$ and the odd bits are all 0 . We set $\sigma[s+1]$ to be the least string which extends $\sigma[s]$ and such that there is a string $\sigma_{R}[s+1]$ with $\sigma_{L}[s]^{\wedge} \tau^{*} \oplus \sigma_{R}[s+1]=\sigma[s+1]$.

(4) If $s=\langle 3, e\rangle$ then let $\sigma[s]=\sigma_{L} \oplus \sigma_{R}[s]$. Similarly to the previous case, we find a finite binary string $\tau$, such that $C\left(X, \sigma_{R}[s]\right)^{\wedge} \tau$ is in $D_{e}\left(\emptyset^{(n)}\right)$. Let $k=|\tau|$. Set $\sigma_{R}[s+1]=\sigma_{R} \hat{\wedge} \tau(0)^{\wedge} 0^{\wedge} \tau(1) \ldots{ }^{\wedge} 0^{\wedge} \tau(k)^{\wedge} 0$ and let $\sigma[s+1]$ be the least string which extends $\sigma[s]$ and such that there is a string $\sigma_{L}[s+1]$ with $\sigma_{L}[s+1] \oplus \sigma_{R}[s+1]=\sigma[s+1]$.

Finally we show that 2-generic degrees can be determined from degrees with images under $\iota$ in the set $\mathcal{I} \mathcal{T}\left(\mathbf{0}_{e}^{\prime}\right)$. Recall that a $\Delta_{2}^{0}$ set is high if its Turing jump computes $\mathbf{0}^{\prime \prime}$ and a Turing degree is high if it contains high set.

Theorem 15. There are high Turing degrees $\mathbf{h}_{1}<\mathbf{0}^{\prime}$ and $\mathbf{h}_{2}<\mathbf{0}^{\prime}$, such that if $\mathbf{x}$ is 2-generic then $\left(\mathbf{h}_{1} \vee \mathbf{x}\right) \wedge\left(\mathbf{h}_{2} \vee \mathbf{x}\right) \equiv_{T} \mathbf{x}$.

Proof. Intuitively $H_{1}$ and $H_{2}$ will be constructed by combining Shoenfield's Jump Inversion Theorem with the attempt to make for every pair of Turing-operators $\Phi, \Psi$ the following set dense:

$$
\left\{\tau \mid \exists x\left(\Phi^{\tau \oplus H_{1}}(x) \neq \Psi^{\tau \oplus H_{2}}(x)\right\}\right) .
$$

Note that for a particular pair of Turing functionals $(\Phi, \Psi)$ this set is $\Sigma_{1}^{0}\left(\emptyset^{\prime}\right)$. Let $G$ be a 2-generic, such that $\Phi^{G \oplus H_{1}}$ and $\Psi^{G \oplus H_{2}}$ are total. If our attempt succeeds then $\Phi^{G \oplus H_{1}} \neq \Psi^{G \oplus H_{2}}$. It will follow from the construction that if our attempt fails and $\Phi^{G \oplus H_{1}}=\Psi^{G \oplus H_{2}}=X$ then $X \leq_{T} G$. 
Using $\emptyset^{\prime}$ as oracle, we build sequences $\mu_{s}$ and $\lambda_{s}$ so that $H_{1}=\bigcup_{s} \mu_{s}$ and $H_{2}=$ $\bigcup_{s} \lambda_{s}$. Let $\left\{C_{s}\right\}_{s<\omega}$ be a computable in $\emptyset^{\prime}$ c.e. approximation of $\emptyset^{\prime \prime}$. For every $s$ let $c_{s}$ be the least element in $C[s] \backslash C[s-1]$. Stages $s$, such that $C_{s}\left\lceil c_{s}+1=C\left\lceil c_{s}+1\right.\right.$ will be called true stages. There are infinitely many true stages.

To every stage $s$ we associate the set of possible extensions of a string $\sigma$ at stage $s$ to be

$$
P_{s}(\sigma)=\left\{\tau \succeq \sigma \mid \forall c, x\left(c<c_{s} \&\langle c, x\rangle \geq|\sigma| \Rightarrow \tau(\langle c, x\rangle)=C_{s}(c)\right)\right\} .
$$

Note that for every $s$ and every $\sigma$ the set $P_{s}(\sigma)$ is computable, although not uniformly computable. If at every stage $s$ we respect that $\mu_{s+1} \in P_{s}\left(\mu_{s}\right)$ and $\lambda_{s+1} \in P_{s}\left(\lambda_{s}\right)$ then both sets $H_{1}$ and $H_{2}$ will be high, by the limit lemma: $C(c)=\lim _{x} H_{i}(\langle c, x\rangle)$. To see this in detail, fix $c$ and let $s$ be larger than $c$ and the first stage $s_{0}$, such that $C_{s_{0}}\lceil c+1=C\lceil c+1$. Then for every $x$, such that $\langle c, x\rangle \geq\left|\mu_{s}\right|$, we will have that $H_{1}(\langle c, x\rangle)=C(c)$. Similarly for every $x$, such that $\langle c, x\rangle \geq\left|\lambda_{s}\right|$, we will have that $H_{2}(\langle c, x\rangle)=C(c)$. We also note that if $s$ is a true stage then for every $t>s$ we have that $\mu_{t} \in P_{s}\left(\mu_{s}\right)$ and similarly $\lambda_{t} \in P_{s}\left(\lambda_{s}\right)$.

Now let $\left\langle\Phi_{e}, \Psi_{e}, \sigma_{e}\right\rangle$ be a computable listing of all triples of Turing operators $\Phi_{e}$ and $\Psi_{e}$ and finite binary string $\sigma_{e}$, called requirements. Initially all requirements are unsatisfied. Set $\mu_{0}=\lambda_{0}=\emptyset$. At stage $s+1$ we have constructed $\mu_{s}$ and $\lambda_{s}$.

For every unsatisfied requirement with index $e<s$ in turn we ask the question "Does there exists a string $\tau \succeq \sigma_{e}$ and strings $\mu \in P_{s}\left(\mu_{s}\right), \lambda \in P_{s}\left(\lambda_{s}\right)$ and a natural number $x$, such that:

$$
\Phi_{e}^{\tau \oplus \mu}(x) \downarrow \neq \Psi_{e}^{\tau \oplus \lambda}(x) \downarrow ?
$$

This question is computable in $\emptyset^{\prime}$. Let $e$ be the index of the least unsatisfied requirement for which the answer is "yes". Find such $\mu$ and $\lambda$ of length larger than the length of $\mu_{s}$ and $\lambda_{s}$ respectively and set $\mu_{s+1}=\mu$ and $\lambda_{s+1}=\lambda$. Declare the requirement with index $e$ satisfied. If the answer for all requirements is "no" then we extend $\mu_{s}$ to $\mu_{s+1} \in P_{s}\left(\mu_{s}\right)$ of length larger than $\left|\mu_{s}\right|$ and $\lambda_{s}$ to $\lambda_{s+1} \in P_{s}\left(\lambda_{s}\right)$ of length larger than $\left|\lambda_{s}\right|$.

Now let $\Psi$ and $\Phi$ be, such that the set $S=\left\{\tau \mid \exists x\left(\Phi^{\tau \oplus H_{1}}(x) \neq \Psi^{\tau \oplus H_{2}}(x)\right)\right\}$ is not dense. Let $\sigma$ be a finite binary string with no extension in $S$. Let $e$ be the index of $\langle\Phi, \Psi, \sigma\rangle$. Let $s+1>e$ be a stage, such that all requirements with indices less that $e$ that ever get satisfied are satisfied and such that $s+1$ is a true stage. At stage $s+1$ the answer to the question for the requirement $e$ is "no". We claim that for any set $G \succeq \sigma$, if $\Phi^{G \oplus H_{1}}$ and $\Psi^{G \oplus H_{2}}$ are total and equal to $X$ then $X$ is computable from $G$. Indeed, to compute $X(x)$, find the least $\mu \in P_{s}\left(\mu_{s}\right)$ and $\lambda \in P_{s}\left(\lambda_{s}\right)$, such that $\Phi^{G \oplus \mu}(x) \downarrow=\Psi^{G \oplus \lambda}(x) \downarrow$. Such a pair exists, because $s+1$ is a good stage and hence $H_{1}$ and $H_{2}$ are infinite paths in the sets $P_{s}\left(\mu_{s}\right)$ and $P_{s}\left(\lambda_{s}\right)$ respectively, and because $\Phi^{G \oplus H_{1}}=\Psi^{G \oplus H_{2}}$. If $X(x) \neq \Phi^{G \oplus \lambda}(x)$, there would be a natural number $n$, such that $\Phi^{G \oplus H_{1}\lceil n}(x) \downarrow \neq \Phi^{G \oplus \lambda}(x)$. But then $H_{1}\left\lceil n\right.$ and $\mu_{s}$ would have a common extension $\mu^{*}$ in $P_{s}\left(\mu_{s}\right)$ and so the answer of the question would be "yes". So, $X(x)=\Phi^{G \oplus \lambda}(x)$.

Definition 5. An indexing of the total $\Delta_{n+1}^{0}$ sets will be any indexing of the sequence $\left\{X_{e} \oplus \overline{X_{e}}\right\}_{e<\omega}$, where $X_{e}=\{e\}^{\emptyset^{(n)}}$ if this is a total $\{0,1\}$-valued functions and $X_{e}=\emptyset$ otherwise.

Theorem 16. Every set of basic indexing parameters defines an indexing of the total $\Delta_{3}^{0}$ sets. 
Proof. Let $\vec{p}$ be basic indexing parameters with a model of true arithmetic $\mathcal{M}$. By Theorem 11 let $\psi^{\mathbf{0}_{e}^{\prime}}$ be a definable from $\vec{p}$ indexing of the $\mathcal{I T}\left(\mathbf{0}_{e}^{\prime}\right)$ total sets. We use $\vec{p}$ to define an indexing $\delta^{\mathbf{0}_{e}^{\prime}}$ of the sequence $\left\{X_{e} \oplus \overline{X_{e}}\right\}_{e<\omega}$, where $X_{e}=\{e\}^{\emptyset^{\prime \prime}}$ if this is a total $\{0,1\}$-valued functions and $X_{e}=\emptyset$ otherwise.

Let $\mathbf{e}$ be an enumeration degree and $e$ be a natural number, such that $\mathbf{e}=e^{\mathcal{M}}$. We determine $\delta^{\mathbf{0}_{e}^{\prime}}(\mathbf{e})$ according to the following cases:

Case 1: In $\mathcal{M}$ the statement " $X_{e}$ belongs to a 2-generic degree." is true. We use Theorem 15 to find high sets $H_{1}$ and $H_{2}$, such that $d_{T}\left(X_{e}\right)$ is the greatest lower bound of the degrees $d_{T}\left(X_{e} \oplus H_{1}\right)$ and $d_{T}\left(X_{e} \oplus H_{2}\right)$. Next we note that the set $X_{e} \oplus H_{1}$ is computable from $\emptyset^{\prime \prime}$ and hence its degree belongs to the interval $\left[d_{T}\left(H_{1}\right), d_{T}\left(H_{1}\right)^{\prime}\right]$. Thus, in $\mathcal{M}$ we can search for the least natural number $i$, such that $H_{1} \oplus X_{e} \equiv_{T} Z_{i}$, where $\left\{Z_{i} \oplus \overline{Z_{i}}\right\}_{i<\omega}$ is the sequence of all total $\mathcal{I} \mathcal{T}\left(\mathbf{0}_{e}^{\prime}\right)$ sets indexed by $\psi^{\mathbf{0}_{e}^{\prime}}$. Similarly in $\mathcal{M}$ we can search for the least natural number $j$, such that $H_{2} \oplus X_{e} \equiv_{T} Z_{j}$. We define $\delta^{\mathbf{0}_{e}^{\prime}}(\mathbf{e})=\psi^{\mathbf{0}_{e}}\left(i^{\mathcal{M}}\right) \wedge \psi^{\mathbf{0}_{e}}\left(j^{\mathcal{M}}\right)$.

Case 2: In $\mathcal{M}$ the statement " $X_{e}^{\prime} \leq_{T} \emptyset^{\prime \prime}$ " is true. Then we use Proposition 2 to find in $\mathcal{M}$ the $\Delta_{3}$ indices $i_{1}, i_{2}, i_{3}$ and $i_{4}$ of 2 -generic sets $G_{1}, G_{2}, G_{3}$ and $G_{4}$, such that $d_{T}\left(X_{e}\right)=\left(d_{T} G_{1} \vee d_{T}\left(G_{2}\right)\right) \wedge\left(d_{T} G_{3} \vee d_{T}\left(G_{4}\right)\right)$. We use Case 1 to determine $\delta^{\mathbf{0}_{e}^{\prime}}\left(i_{j}^{\mathcal{M}}\right)$ for $j=1,2,3,4$ and set $\delta^{\mathbf{0}_{e}^{\prime}}(\mathbf{e})=\left(\delta^{\mathbf{0}_{e}^{\prime}}\left(i_{1}^{\mathcal{M}}\right) \vee \delta^{\mathbf{0}_{e}^{\prime}}\left(i_{2}^{\mathcal{M}}\right)\right) \wedge\left(\delta^{\mathbf{0}_{e}^{\prime}}\left(i_{3}^{\mathcal{M}}\right) \vee \delta^{\mathbf{0}_{e}^{\prime}}\left(i_{4}^{\mathcal{M}}\right)\right)$.

Case 3: Otherwise, we use Proposition 1 to find in $\mathcal{M}$ the $\Delta_{3}$ indices $i_{1}, i_{2}, i_{3}$ and $i_{4}$ of sets $Y_{1}, Y_{2}, Y_{3}$ and $Y_{4}$ whose jumps are computable in $\emptyset^{\prime \prime}$ and such that $d_{T}\left(X_{e}\right)=\left(d_{T} Y_{1} \vee d_{T}\left(Y_{2}\right)\right) \wedge\left(d_{T} Y_{3} \vee d_{T}\left(Y_{4}\right)\right)$. We use Case 2 to determine $\delta^{\mathbf{0}_{e}^{\prime}}\left(i_{j}^{\mathcal{M}}\right)$ for $j=1,2,3,4$ and set $\delta^{\mathbf{0}_{e}^{\prime}}(\mathbf{e})=\left(\delta^{\mathbf{0}_{e}^{\prime}}\left(i_{1}^{\mathcal{M}}\right) \vee \delta^{\mathbf{0}_{e}^{\prime}}\left(i_{2}^{\mathcal{M}}\right)\right) \wedge\left(\delta^{\mathbf{0}_{e}^{\prime}}\left(i_{3}^{\mathcal{M}}\right) \vee \delta^{\mathbf{0}_{e}^{\prime}}\left(i_{4}^{\mathcal{M}}\right)\right)$.

\section{Applichtions}

Let $\vec{p}$ be basic indexing parameters. In Section 3 we showed that $\vec{p}$ define an indexing $\delta$ of the total $\Delta_{2}^{0}$ sets. In Section 4 we extended this to show that $\vec{p}$ defines firs a $\mathbf{0}_{e}^{\prime}$-basic indexing $\varphi^{\mathbf{0}_{e}^{\prime}}$ and then an indexing $\psi^{\mathbf{0}_{e}^{\prime}}$ of the total $\mathcal{I T}\left(\mathbf{0}_{e}^{\prime}\right)$ sets. Finally in Section 5 we extended this result one step further to show that $\vec{p}$ define an indexing $\delta^{\mathbf{0}_{e}^{\prime}}$ of the total $\Delta_{3}^{0}$ sets. We can continue in this fashion: from $\vec{p}$ and $\delta^{\mathbf{0}_{e}^{\prime}}$ we can define a $\mathbf{0}_{\mathbf{e}}^{\prime \prime}$-basic indexing $\varphi^{\mathbf{0}_{\mathbf{e}}^{\prime \prime}}$ and then an indexing $\psi^{\mathbf{0}_{\mathbf{e}}^{\prime \prime}}$ of all total sets in $\mathcal{I} \mathcal{T}\left(\mathbf{0}_{\mathbf{e}}^{\prime \prime}\right)$. Then we use the methods of Section 5 with Theorem 15 relativized to $\mathbf{0}^{\prime}$ to define an indexing $\delta^{\mathbf{0}_{\mathbf{e}}^{\prime \prime}}$ of the total $\Delta_{4}^{0}$ sets. Iterating further we obtain our main result.

Theorem 17. Let $\vec{p}$ be basic indexing parameters. For every natural number $n, \vec{p}$ define an indexing of the total $\Delta_{n}^{0}$ sets.

Combining this result with Theorem 4 we obtain the following result:

Theorem 18. Every set of parameters $\vec{p}$ that define a basic indexing is an automorphism base of $\mathcal{D}_{e}$.

Proof. Let $\vec{p}$ be parameters that define a basic indexing and let $\pi: \mathcal{D}_{e} \rightarrow \mathcal{D}_{e}$ be an automorphism of the enumeration degrees which fixes all element of $\vec{p}$. Let $\mathcal{M}$ be the standard model of arithmetic associated with $\vec{p}$. For every natural number $n, n^{\mathcal{M}}$ is definable from $\vec{p}$, hence $\pi\left(n^{\mathcal{M}}\right)=n^{\mathcal{M}}$. By Theorem 17 using $\vec{p}$ we can define an indexing $\delta^{\mathbf{0}_{e}^{(4)}}$ of the sequence $\left\{X_{e} \oplus \overline{X_{e}}\right\}_{e<\omega}$, where $X_{e}=\{e\}^{\emptyset^{(5)}}$ if this is a total $\{0,1\}$-valued function and $X_{e}=\emptyset$ otherwise. So if $\mathbf{x} \leq \mathbf{0}_{e}^{(5)}$ is 
a total enumeration degree then there is an $e$, such that $\mathbf{x}=\delta^{\mathbf{0}_{e}^{(4)}}\left(e^{\mathcal{M}}\right)$ and so $\pi(\mathbf{x})=\pi\left(\delta^{\mathbf{0}_{e}^{(4)}}\left(e^{\mathcal{M}}\right)\right)=\delta^{\mathbf{0}_{e}^{(4)}}\left(\pi\left(e^{\mathcal{M}}\right)\right)=\delta^{\mathbf{0}_{e}^{(4)}}\left(e^{\mathcal{M}}\right)=\mathbf{x}$. In other words $\pi$ fixes all total degrees below $\mathbf{0}_{e}^{(5)}$, which by Theorem 4 is an automorphism base for $\mathcal{D}_{e}$. It follows that $\pi$ is the identity.

Finally we give a connection between local structures and the global structure of the enumeration degrees.

Theorem 19. The rigidity of the global structure of the enumeration degrees is implied by any of the following:

(1) The rigidity of $\mathcal{D}_{e}\left(\leq \mathbf{0}_{e}^{\prime}\right)$;

(2) The rigidity of $\mathcal{D}_{T}\left(\leq \mathbf{0}_{T}^{\prime}\right)$;

(3) The rigidity of the computably enumerable Turing degrees.

Proof. Every automorphism of $\mathcal{D}_{e}$ induces an automorphism of $\mathcal{D}_{e}\left(\leq \mathbf{0}_{e}^{\prime}\right), \mathcal{D}_{T}\left(\leq \mathbf{0}_{T}^{\prime}\right)$ and the computably enumerable degrees, because the enumeration jump, the total enumeration degrees below $\mathbf{0}_{e}^{\prime}$ and the image of the computably enumerable degrees under the standard embedding of $\mathcal{D}_{T}$ into $\mathcal{D}_{e}$ are each first order definable in $\mathcal{D}_{e}$ by Theorem 1, Theorem 3 and Theorem 5 respectively. If we assume that any of these structures is rigid then any automorphism of $\mathcal{D}_{e}$ would fix all total enumeration degrees below $\mathbf{0}_{e}^{\prime}$. This is obvious for $\mathcal{D}_{e}\left(\leq \mathbf{0}_{e}^{\prime}\right)$ and $\mathcal{D}_{T}\left(\leq \mathbf{0}_{T}^{\prime}\right)$ and follows from Corollary 1 for the computably enumerable Turing degrees. From Theorem 6 it follows that there are total enumeration degrees in $\mathcal{D}_{e}\left(\leq \mathbf{0}_{e}^{\prime}\right)$ that define a basic indexing. By the previous theorem they are an automorphism base for $\mathcal{D}_{e}$. Thus it would follow that the only possible automorphism of $\mathcal{D}_{e}$ is the identity.

Our investigations naturally lead to the following open questions:

Question 1. Does rigidity for any one of the local structures imply rigidity for either of the other local structures? In particular, is every enumeration degree in $\mathcal{D}_{e}\left(\leq \mathbf{0}_{e}^{\prime}\right)$ definable in $\mathcal{D}_{e}\left(\leq \mathbf{0}_{e}^{\prime}\right)$ from total degrees?

Question 2. Does rigidity for any of the local structures or $\mathcal{D}_{e}$ imply rigidity for the structure of the Turing degrees?

\section{Determining the LOW EnUMERATion Degrees From the LOW CO-D.C.E. AND THE CO-C.E. DEGREES}

This section is devoted to the proof of Theorem 9. We wish to prove that for every pair of $\Delta_{2}^{0}$ enumeration degrees $\mathbf{x}, \mathbf{y}$, such that $\mathbf{x}^{\prime}=\mathbf{0}_{e}^{\prime}$ and $\mathbf{y} \not \mathbf{x}$ there are $\Delta_{2}^{0}$ enumeration degrees $\mathbf{g}_{i}, \Pi_{1}^{0}$ enumeration degrees $\mathbf{a}_{i}$ and low co-d.c.e. enumeration degrees $\mathbf{c}_{i}, \mathbf{b}_{i}$ for $i=1,2$, such that:

(1) $\mathbf{g}_{i}$ is the least element below $\mathbf{a}_{i}$ which joins $\mathbf{b}_{i}$ above $\mathbf{c}_{i}$;

(2) $\mathbf{x} \leq \mathbf{g}_{1} \vee \mathbf{g}_{2}$

(3) $\mathbf{y} \not \mathbf{g}_{1} \vee \mathbf{g}_{2}$.

In the previous sections we used the notation $\Phi(A)$ to denote the image of a set $A$ under the enumeration operator $\Phi$, as it was useful to have different ways of representing the relations "c.e. in" and " $\leq e$ ". From now one we will adopt the notation $\Phi^{A}$ for $\Phi(A)$, the result of applying the enumeration operator $\Phi$ to $A$.

We will use a characterization of the low sets due to Cooper and McEvoy [10].

Proposition 3. A set $X$ is low if an only if there is a $\Delta_{2}^{0}$ approximation $\{X[s]\}_{s<\omega}$ to $X$ with the following property: for every enumeration operator $W$, approximated 
by its standard c.e. approximation $\{W[s]\}_{s<\omega}$, and every natural number $x$ if $x \in$ $W[s]^{X[s]}$ at infinitely many stages $s$ then $x \in W^{X}$.

Let $X$ be a low $\Delta_{2}^{0}$ set with a low $\Delta_{2}^{0}$ approximation $\{X[s]\}_{s<\omega}$. Fix $Y \not \Varangle_{e} X$ to be a $\Delta_{2}^{0}$ set with a $\Delta_{2}^{0}$ approximation $\{Y[s]\}_{s<\omega}$. We will construct approximations to sets $A_{i}, B_{i}, C_{i}$ and $G_{i}$ for $i=1,2$ to witness the statement of the theorem for $X$ and $Y$. To ensure that $A_{i}$ is $\Pi_{1}^{0}$, the approximation $\left\{A_{i}[s]\right\}_{s<\omega}$ will have $A_{i}[0]=\mathbb{N}$ and for all $s, A_{i}[s] \supseteq A_{i}[s+1]$. To ensure that any $E \in\left\{C_{1}, C_{2}, B_{1}, B_{2}\right\}$ is co-d.c.e, its approximation will have the property $E[0]=\mathbb{N}$ and for every natural number $n$ there will be at most one stage $s$, such that $n \in E[s] \backslash E[s+1]$. In other words if the number $n$ leaves the approximation to $E$ then it can return to it, but once it returns, it can never leave again. Finally, the sets $G_{i}, i=1,2$ will be $\Delta_{2}^{0}$ as their approximations will satisfy: $G_{i}[0]=\mathbb{N}$ and for every $n, \lim _{s} G_{i}[s](n)$ exists.

Our construction must be designed so that the following requirements are met.

(1) $\Lambda_{i}: G_{i}=\Lambda_{i}^{A_{i}}$, ensuring that $G_{i}$ is reducible to $A_{i}$.

(2) $\Gamma_{i}: C_{i}=\Gamma_{i}^{B_{i}, G_{i}}$, ensuring that $B_{i}$ joins $G_{i}$ above $C_{i}$.

(3) $\Omega: X=\Omega^{G_{1}, G_{2}}$, ensuring that $X$ is enumerable from $G_{1} \oplus G_{2}$.

(4) Next we ensure that out of all sets with the first two properties above $G_{i}$ has the least enumeration degree. Let $\left(\Theta_{e}, \Phi_{e}\right)_{e<\omega}$ be a listing of all pairs of enumeration operators. For every $e$ we have the requirement:

$\mathcal{R}_{e}^{i}$ : If $\Theta_{e}^{\Phi_{e}^{A_{i}}, B_{i}}=C_{i}$ then there is an enumeration operator $\Delta_{e}$, such that $G_{i}=\Delta_{e}^{\Phi_{e}^{A} i}$.

(5) To ensure that $Y$ is not enumerable from $G_{1} \oplus G_{2}$, we have the following list of requirements. Let $\left(\Psi_{e}\right)_{e<\omega}$ be a listing of all enumeration operators. For every $e$ we have the requirement: $\mathcal{Q}_{e}: \Psi_{e}^{G_{1}, G_{2}} \neq Y$.

(6) Finally to ensure that $B_{i}$ and $C_{i}$ are low we use Proposition 3. Let $\left\{\Upsilon_{i}\right\}_{i<\omega}$ be a listing of all enumeration operators. For every $e=\langle i, x\rangle$, we have the lowness requirement

$$
\mathcal{L}_{e}: \text { If } x \in \Upsilon_{i}^{B_{1}, B_{2}, C_{1}, C_{2}} \text { at infinitely many stages then } x \in \Upsilon_{i}^{B_{1}, B_{2}, C_{1}, C_{2}} \text {. }
$$

7.1. Description of strategies. To every requirement we attach a strategy, designed to work towards its satisfaction. The construction will then run in stages and every stage will consist of activating different strategies. The choice of which strategy is activated will depend on the actions of higher priority strategies that are activated at the current stage and the approximations to the given sets. For any given operator $W$ that we approximate we will assume that if $x \in W^{Z}[s]$ then $x<s$ and $x \in W^{Z \uparrow s}$. We start with the description of the strategies $\Lambda_{i}, \Gamma_{i}$ and $\Omega$, which are called global strategies and will be activated at the beginning of every stage. The goal of each is to construct an enumeration operator, the name of which coincides with theirs.

The $\Lambda$-strategies. The strategy $\Lambda_{i}$ uses markers to build its operator. If currently $n \in G_{i} \backslash \Lambda_{i}^{A_{i}}$ then the strategy assigns to $n$ a new marker, $\lambda_{i}(n)$, chosen fresh (larger than twice the largest number mentioned so far), and enumerates a new axiom $\left\langle n, A_{i}\left\lceil\lambda_{i}(n)\right\rangle\right.$ in the operator $\Lambda_{i}$. Here $A\lceil x$ is meant to be all numbers that are in $A$ and are less than or equal to $x$. During the construction, whenever we extract $n$ from $G_{i}$, we will automatically also extract the marker $\lambda_{i}(n)$ from $A_{i}$, so that we always have $G_{i}(n)=\Lambda_{i}^{A_{i}}(n)$. 
The $\Gamma$-strategies. The $\Gamma_{i}$-strategy also assigns to every natural number $c$ a marker $\gamma(c)$. However, $c$ will also have an additional marker $g_{i}(c)$, controlled by the $\mathcal{R}^{i}$ strategies. When activated, the strategy ensures that if currently $c \in C_{i}$ then there is a valid axiom for $c$ in $\Gamma_{i}$ of the form $\left\langle c, B_{i}\left\lceil\gamma_{i}(c), G_{i}\left\lceil g_{i}(c)\right\rangle\right.\right.$. During the construction we will permanently extract a number $c$ from $C_{i}$ if we can be sure that all axioms for $c$ enumerated so far in $\Gamma_{i}$ can never be valid again.

The $\Omega$-strategy. For every element $x$ the strategy defines an $\Omega$-marker $\omega(x)$. We will distinguish between the current $\Omega$-marker $\omega(x)$ and previously defined $\Omega$ markers $o(x)$. The marker $\omega(x)$ will always be chosen as a fresh number, so that $\omega(x) \in G_{1} \cap G_{2}$. Every axiom enumerated in $\Omega$ for $x$, say $\left\langle x, D_{1}, D_{2}\right\rangle$ will include some marker $o(x)$ in both $D_{1}$ and $D_{2}$. Thus extracting $o(x)$ from either oracle set will invalidate the axiom. The $\Omega$-strategy makes sure that at every stage $s$ we have $\Omega^{G_{1}, G_{2}}[s]=X[s]$ by enumerating axioms for numbers in $X[s]$ and extracting numbers from $G_{1}$ or $G_{2}$ to invalidate axioms for numbers not in $X[s]$.

Now we turn to the two more difficult requirements, $\mathcal{R}_{e}^{i}$ and $\mathcal{Q}_{e}$. We will handle them using a tree of strategies. We will denote $\mathcal{R}_{e}^{i}$ strategies by $\alpha$, and $\mathcal{Q}_{e}$ strategies by $\beta$. We order the requirements by priority linearly as follows:

$$
\mathcal{R}_{0}^{1}<\cdots<\mathcal{R}_{e}^{1}<\mathcal{R}_{e}^{2}<\mathcal{Q}_{e}<\mathcal{R}_{e+1}^{1} \cdots
$$

Strategies of level $n$ in the tree of strategies are assigned the $n$-th requirement in the priority ordering. The branching in the tree is determined by the outcomes of the strategies. The lexicographical ordering of nodes in the tree induces a priority ordering of the strategies.

The $\mathcal{R}$-strategies. Suppose that $\alpha$ is working towards satisfying the requirement $\mathcal{R}_{e}^{1}$. We will drop the subscript $e$ in the discussion below. The strategy will have three possible outcomes: $f<_{L} i<_{L} w$. The outcome $f$ will signify that the strategy was successful in diagonalizing $\Theta^{\Phi_{1}^{A}, B_{1}}$ against $C_{1}$ : a number $c$ was found, such that $c \in \Theta^{\Phi_{1}^{A}, B_{1}} \backslash C_{1}$. We explain below how this outcome can be reached, however let us first think about how such diagonalization can be preserved at further stages. The strategy would like to prevent certain numbers from leaving the approximations to $A_{1}$ and $B_{1}$ to keep $c \in \Theta^{\Phi_{1}^{A}, B_{1}}$. Restraints on $B_{1}$ will be achieved through initialization of lower priority strategies and the general rule that every time a strategy restarts its work, it deals with fresh numbers, not used so far. Restraints on $A_{1}$ are more difficult, as changes in $X$ might require changes in $G_{1}$ and therefore changes in $A_{1}$. In order to preserve $A_{1}$, the strategy will have to set things up as follows.

The strategy $\alpha$ keeps a parameter $s_{0}(\alpha)$, where it records the first stage at which it is activated since the last time it was initialized. The strategy $\alpha$ first leaves room for the $\Omega$-strategy to successfully define its operator on all numbers less than $s_{0}(\alpha)$. Every time $X\left\lceil s_{0}(\alpha)\right.$ changes, $\alpha$ is restarted. Thus $\alpha$ can assume in its later work that $X\left\lceil s_{0}(\alpha)\right.$ does not change and no number less than $\omega\left(s_{0}(\alpha)\right)$ will be forced out of either $G_{i}$ or $A_{i}$. We denote by $s_{1}(\alpha)$ the least stage, such that $\alpha$ is not restarted after it. At stage $s_{1}(\alpha)$ the strategy selects a threshold $d_{\alpha}$ as the least number which is greater than $\omega\left(s_{0}(\alpha)\right)$ and in $G_{2}$. The design of the $\Omega$-strategy will then ensure that $\alpha$ can from now on at any time preserve $G_{1}$ (and so $A_{1}$ ) on elements greater than or equal to $d_{\alpha}$ by extracting $d_{\alpha}$ from $G_{2}$. Once all of this is set up the strategy proceeds to its actual work. 
First $\alpha$ looks at the largest number $l$ such that $C_{1} \uparrow l \subseteq \Theta^{\Phi^{A_{1}}, B_{1}}$. We will call this the length of inclusion. Note that this notion is different from the usual length of agreement, but it plays a similar role: a bounded length of inclusion guarantees that the two sets are different. If at the current stage this length is greater than it was at previous stages, we say that the stage is expansionary. The strategy will have outcome $w$ at stages that are not expansionary. If from some stage onwards the strategy always has outcome $w$, then $\Theta^{\Phi^{A_{1}}, B_{1}} \neq C_{1}$ as there is some number $y \in C_{1}$, such that $y \notin \Theta^{\Phi^{A_{1}}, B_{1}}$.

At expansionary stages $\alpha$ starts working on its operator $\Delta_{\alpha}$. It scans numbers one by one, trying to enumerate axioms for $n \in G_{1}$ and checking that there are no valid axioms for numbers $n \notin G_{1}$. If all numbers are scanned then the outcome will be $i$. If this is the true outcome at infinitely many stages then we will argue that $\Delta_{\alpha}^{\Phi^{A_{1}}}=G_{1}$. If on the other hand $\alpha$ discovers a number $n \in \Delta_{\alpha}^{\Phi^{A_{1}}} \backslash G_{1}$ then this number will be used for a successful diagonalization and $\alpha$ will have outcome $f$. To explain the mechanism behind this, we need to go into the details of how $\Delta_{\alpha}$ is constructed.

The strategy $\alpha$ has a list of chits $C_{\alpha}$ containing only numbers larger than $s_{1}(\alpha)$. Every natural number $n \in G_{1}$ is assigned a chit $c(n) \in C_{\alpha}$. When this chit is assigned it is a fresh number, the strategy $\Gamma_{1}$ has not enumerated axioms for it. We set its second marker $g_{1}(c(n))$ to $n$. Thus every $\Gamma_{1}$-axiom for $c(n)$ that will be enumerated from now on will use $G_{1} \uparrow n$. The number $c(n)$ will remain the chit for $n$ until (if ever) we see a change in $G_{1} \uparrow n$. If we see such a change, we will pick a fresh chit for $n$. The chits are used to define axioms for $n$ in $\Delta_{\alpha}$. If $n \in G_{1}$ has been assigned the chit $c(n)$ and $c(n) \in \Theta^{\Phi_{1}^{A_{1}}, B_{1}}$ via an axiom $\left\langle c(n), D_{\Phi}, D_{B}\right\rangle$, then the strategy enumerates the axiom $\left\langle n, D_{\Phi}\right\rangle$ in $\Delta_{\alpha}$. We say that this axiom is defined using the triple $\left\langle c, D_{\Phi}, D_{B}\right\rangle$.

Suppose that later on $n \in \Delta_{\alpha}^{\Phi_{e}^{A_{1}}} \backslash G_{1}$ and the valid axiom for $n$ in $\Delta_{\alpha}$ is defined via the triple $\left\langle c(n), D_{\Phi}, D_{B}\right\rangle$. We enumerate $D_{B}$ back in $B_{1}$, as lower priority strategies might have extracted numbers out of $B_{1}$, ensuring that $c(n) \in \Theta^{\Phi^{A_{1}}, B_{1}}$. We then extract $c(n)$ from $C_{1}$ and achieve the desired diagonalization. To preserve it at further stages we initialize all lower priority strategies and extract $d_{\alpha}$ from $G_{2}$, as discussed above.

Finally we must check that this change in $C_{1}$ will not injure the strategy $\Gamma_{1}$. Note that as $n \notin G_{1}$, any axiom defined for $c(n)$ until this change occurred will be invalid. To ensure that $\Gamma_{1}$ is correct on $c(n)$ we must invalidate all axioms defined after $n$ left the approximation to $G_{1}$. We do so by extracting the corresponding $\gamma_{1}(c(n))$ markers. It follows that they are larger than max $D_{B}$ and so will not interfere with the restraint on $B_{1}$. As long as $\alpha$ is not initialized or restarted this will keep $\Gamma_{1}$ correct. If $\alpha$ does get restarted, we will enumerate the number $c(n)$ back in $C_{1}$.

The $\mathcal{Q}$-strategies. Let $\beta$ be a $\mathcal{Q}$-strategy working on the requirement $\mathcal{Q}_{e}$. We drop the index $e$ in the discussion below. The strategy $\beta$ will have one outcome $f$, but it will cancel all lower priority strategies whenever it acts. The goal of the strategy is to construct an operator $\Xi$, such that $\Xi^{X}=\Psi^{G_{1}, G_{2}}$ on all numbers up to and including the first difference between the sets $\Psi^{G_{1}, G_{2}}$ and $Y$. As $Y \not \mathbb{E}_{e} X$, it will follow that this strategy will eventually find a permanent finite difference between $\Psi^{G_{1}, G_{2}}$ and $Y$. 
Just like the $\mathcal{R}$-strategies, $\beta$ first leaves space for the $\Omega$-strategy to define its operator correctly by keeping a parameter $s_{0}(\beta)$, the first activation after the previous initialization. Whenever $G_{1}\left\lceil s_{0}(\beta)\right.$ or $G_{2}\left\lceil s_{0}(\beta)\right.$ change the strategy is restarted. The last stage when $\beta$ is restarted is recorded in $s_{1}(\beta)$. From this stage onwards it assumes that $G_{1}\left\lceil s_{0}(\beta)\right.$ and $G_{2}\left\lceil s_{0}(\beta)\right.$ are fixed.

At further stages the strategy enumerates axioms in $\Xi$ for all elements $x$, such that $x \in \Psi^{G_{1}, G_{2}}$ and $x \leq l$, where $l$ is the least difference between $Y$ and $\Psi^{G_{1}, G_{2}}$. It finds the least valid axiom $\left\langle x, D_{1}, D_{2}\right\rangle \in \Psi$. Let $D_{X}$ be the set of all $n \in X$, such that $o(n) \in D_{1} \cup D_{2}$. Notice that as long as $D_{X} \subseteq X$, we will also have that $D_{1} \oplus D_{2} \subseteq G_{1} \oplus G_{2}$ and hence $x \in \Psi^{G_{1}, G_{2}}$. The strategy enumerates the axiom $\left\langle x, D_{X}\right\rangle$ in $\Xi$.

The $\Delta_{2}^{0}$ nature of $X$ allows for the following possibility. A number $n \in D_{X}$ exits the approximation to $X$, which in turn forces the $\Omega$ strategy to extract a number from $D_{1}$ or $D_{2}$ out of $G_{1}$ or $G_{2}$ respectively. If this invalidates all axioms for $x$ in $\Psi$, at the next visit of $\beta$ we will have $x \notin \Psi^{G_{1}, G_{2}}$. If meanwhile the number $n$ has re-entered $X$, we are left with a mistake in $\Xi$. We can fix this by re-enumerating $D_{1}$ and $D_{2}$ back in $G_{1}$ and $G_{2}$ respectively. We implement this by attaching a list $P$ of promises to $\beta$. A new promise is made for every axiom enumerated in $\Xi$. It has the form $\left\langle D_{X}, D_{G_{1}}, D_{G_{2}}\right\rangle$ and means that we promise: if $D_{X} \subseteq X$ then $D_{G_{i}} \subseteq G_{i}$ for $i=1,2$. At every stage after $s_{1}(\alpha)$ the strategy ensures that it is keeping all its promises. It does that by changing the approximation to $G_{1} \oplus G_{2}$ (only on numbers greater than $\left.s_{0}(\beta)\right)$ according to the promise list and the current approximation to the set $X$.

The actions of $\beta$ ensure that at every stage $s$ we have that $\Xi^{X}$ agrees with $Y$ on the largest initial segment such that $\Psi^{G_{1}, G_{2}}$ agrees with $Y$. If this initial segment is unbounded then for every $x$ there would be infinitely many $s$, such that $\Xi^{X}(x)[s]=$ $Y(x)[s]$. By our choice of low approximation for $X$ and $\Delta_{2}^{0}$ approximation to $Y$, it would follow that $\Xi^{X}(x)=Y(x)$. As $Y \not_{e} X$, the initial segment on which $Y$ and $\Psi^{G_{1}, G_{2}}$ agree must be bounded by a least number, say $l$. So there must be some number $x \leq l$ such that at infinitely many stages $s$ we have that $Y[s](x) \neq$ $\Psi^{G_{1}, G_{2}}[s](x)$. Now, if $x \in Y$ it follows immediately that $Y(x) \neq \Psi^{G_{1}, G_{2}}(x)$. If $x \notin Y$ then by construction $x \in \Xi^{X}$ at infinitely many stages, and now again by the lowness of $X, x \in \Xi^{X}$. By the way we design our promise list it will follow that $x \in \Psi^{G_{1}, G_{2}}$.

The $\mathcal{L}$-strategies. Let $e=\langle i, x\rangle$. The $\mathcal{L}_{e}$-strategy will be activated at all stages $s \geq e$. It's goal is to ensure that if $x \in \Upsilon_{i}^{B_{1}, B_{2}, C_{1}, C_{2}}[s]$ at infinitely many stages $s$ then $x \in \Upsilon_{i}^{B_{1}, B_{2}, C_{1}, C_{2}}$. Whenever it sees a valid axiom for $x$ in $\Upsilon_{i}$, it tries to restrain elements in $B_{1}, B_{2}, C_{1}$ and $C_{2}$ in order to keep this axiom valid at all further stages. The $\mathcal{R}$ strategies are the ones that can interfere with this plan. We know, however that an $\mathcal{R}$-strategy $\alpha$ only extracts numbers from $B_{1}, B_{2}, C_{1}$ or $C_{2}$ that are larger than $s_{1}(\alpha)$ and it does this only once: when it implements a diagonalization. So the $\mathcal{L}_{e}$-strategy operates as follows: if at stage $s$ it sees that $x \in \Upsilon_{i}^{B_{1}, B_{2}, C_{1}, C_{2}}[s] \backslash \Upsilon_{i}^{B_{1}, B_{2}, C_{1}, C_{2}}[s-1]$ then it restarts all $\mathcal{R}$-strategies $\alpha$ with current value of the parameter $s_{0}(\alpha)>e$. By restarting an $\mathcal{R}$-strategy $\alpha$ we change the value of its parameter $s_{1}(\alpha)$, but do not change the value of $s_{0}(\alpha)$, hence $\mathcal{L}_{e}$ will have finite impact on the $\mathcal{R}$-strategies. 
7.2. Construction. We combine the ideas described above in a formal construction. We have global strategies: $\Lambda_{0}, \Lambda_{1}, \Gamma_{0}, \Gamma_{1}$ and $\Omega$, the lowness strategies $\mathcal{L}_{e}$ for $e<\omega$ and a tree $T \subseteq\{f, i, w\}^{<\omega}$ of $\mathcal{R}$ and $\mathcal{Q}$ strategies. Every strategy $\alpha$ in the tree $T$ is given higher priority than all strategies that extend it or that are to the right of it in $T$.

To every strategy we attach parameters as follows:

(1) $\Lambda_{i}$ has as parameter the operator that it constructs. It dynamically assigns markers $\lambda_{i}(n)$ to every number $n$.

(2) $\Gamma_{i}$ has as parameter the operator that it constructs. It dynamically assigns markers $\gamma_{i}(c)$ to every number $c$.

(3) $\Omega$ also has it operator and assigns markers $o(x)$ to all natural numbers $x$.

(4) An $\mathcal{L}_{e}$-strategy has a flag $w_{e}$, which is initially 0 and can be turned to 1.

(5) An $\mathcal{R}_{e}^{i}$-strategy $\alpha$ has the following parameters: $s_{0}(\alpha)$, the stage when $\alpha$ started work after being initialized; $s_{1}(\alpha)$ the stage after the last time $\alpha$ is restarted; a threshold $d_{\alpha}$, used to ensure that $G_{i}$ and $A_{i}$ can be preserved; the number $l_{\alpha}$, measuring the length of inclusion at every stage; an operator $\Delta_{\alpha}$; a list of chits $C_{\alpha}$. In addition the strategy will dynamically assign chits $c(n)$ to natural numbers $n$, define their $g_{i}$-markers and record the stage at which this happened in $s_{c(n)}$. When $\alpha$ is initialized all parameters become undefined. When $\alpha$ is restarted all parameters except $s_{0}(\alpha)$ become undefined. In both cases any chit that $\alpha$ extracts from $C_{i}$ is enumerated back in $C_{i}$.

(6) A $\mathcal{Q}_{e}$-strategy $\beta$ has the following parameters: $s_{0}(\beta)$, the $\beta$-true stage after the last initialization; $s_{1}(\beta)$, the $\beta$-true stage after the last restart; a number $l_{\beta}$, recording the first difference between $Y$ and $\Psi_{e}^{G_{1}, G_{2}} ;$ a list of promises $P_{\beta}$; a functional $\Xi_{\beta}$; If $\beta$ is initialized all parameters become undefined. When $\beta$ is restarted all parameters except $s_{0}(\beta)$ become undefined.

\section{Construction:}

At stage 0 we initialize all strategies and set $A_{i}=B_{i}=C_{i}=G_{i}=\mathbb{N}$. At stage $s \geq 0$ all parameters inherit their values from the previous stage unless they are explicitly modified during stage $s$. We start stage $s$ by visiting the global strategies as follows:

Step I: $\Omega$ : Scan all $n<s$.

(1) If $x \in X \backslash \Omega^{G_{1}, G_{2}}$ then let $\omega(x)$ be a fresh number and enumerate in $\Omega$ the axiom $\left\langle x, G_{1}\left\lceil\omega(x), G_{2}\lceil\omega(x)\rangle\right.\right.$ (the axiom is defined with marker $\omega(x)$ ).

(2) If $x \in \Omega^{G_{1}, G_{2}} \backslash X$ then for every valid axiom for $x$ in $\Omega$ defined with marker $o(x)$ : extract $o(x)$ from $G_{1}$ and $\lambda_{1}(o(x))$ from $A_{1}$ if $o(x)$ is even; extract $o(x)$ from $G_{2}$ and $\lambda_{2}(o(x))$ from $A_{2}$ if $o(x)$ is odd.

Step II: $\Lambda_{i}$ : Scan all elements $n<s$. If $n \in G_{i} \backslash \Lambda_{i}^{A_{i}}$ then let $\lambda_{i}(n)$ be a fresh number and enumerate the axiom $\left\langle n, A_{i}\left\lceil\lambda_{i}(n)\right\rangle\right.$ in $\Lambda_{i}$.

Step III: $\Gamma_{i}$ : Scan all $n<s$. If $n \in C_{i} \backslash \Gamma_{i}^{B_{i}, G_{i}}$ then let $\gamma_{i}(n)$ be a fresh number. If $g_{i}(s) \uparrow$ then set $g_{i}(s)=0$. Enumerate in $\Gamma_{i}$ the axiom $\left\langle n, B_{i}\left\lceil\gamma_{i}(n), G_{i}\left\lceil g_{i}(n)\right\rangle\right.\right.$.

Step IV: $\mathcal{L}:$ For al $e=\langle i, x\rangle<s$ do the following:

(1) If $w_{e}=1$ but $x \notin \Upsilon_{i}^{B_{1}, B_{2}, C_{1}, C_{2}}[s]$ then set $w_{e}=0$. 
(2) If $w_{e}=0$ and $x \in \Upsilon_{i}^{B_{1}, B_{2}, C_{1}, C_{2}}[s]$ then set $w_{e}$ to 1 and restart all $\mathcal{R}$ strategies $\alpha$ with $e \leq s_{0}(\alpha)[s]$.

Step V: Construction of $\delta$ : We construct a finite path $\delta[s]$ in $T$. The path $\delta[s]$ is defined inductively. Set $\delta[s]\lceil 0=\emptyset$. Suppose that we have constructed $\delta[s] \uparrow k$. If $k=s$, then set $\delta[s]=\delta[s]\lceil k$. Otherwise, we activate the strategy $\delta[s]\lceil k$. We say that $s$ is a $\delta[s]\lceil k$-true stage. If the strategy ends the stage then $\delta[s]=\delta[s]\lceil k$. Otherwise the strategy produces an outcome $o$, and $\delta[s] \uparrow k+1=\left(\delta[s]\lceil k)^{\wedge} o\right.$. We have three cases depending on the type of the strategy $\delta[s]\lceil k$ :

Case $\mathcal{R}_{e}^{i}$ Suppose that $\delta[s]\left\lceil k\right.$ is an $\mathcal{R}_{e}^{1}$-strategy $\alpha$. Let $s^{-}$be the previous stage at which $\alpha$ was visited and $o^{-}$be the outcome that $\alpha$ had at stage $s^{-}$. (If $\alpha$ has never been visited then $s^{-}=0$ and $\left.o^{-}=w\right)$. Pick the first case which applies:

(1) If $s_{0}(\alpha)$ is not defined then set $s_{0}(\alpha)=s$. End this stage.

(2) If $s_{1}(\alpha)$ is not defined then set $s_{1}(\alpha)=s$ and $d_{\alpha}=\omega\left(s_{0}(\alpha)\right)+1$. Set $C_{\alpha}=\left\{\langle\hat{\alpha}, n\rangle \mid n>s_{1}(\alpha)\right\}$ where $\hat{\alpha}$ is the code of $\alpha$ in some fixed computable coding of all finite binary strings. End this stage.

(3) If $s_{1}(\alpha)$ is defined, but $X\left\lceil s_{0}(\alpha)\right.$ changed at a stage $t$, such that $s_{1}(\alpha)<$ $t \leq s$ then restart $\alpha$. End this stage.

(4) If $o^{-}=f$ then let the outcome be $f$.

(5) Let $l_{\alpha}$ be maximal number $l$ such that $C_{1} \uparrow l \subseteq \Theta_{\alpha}^{\Phi_{\alpha}^{A_{1}}, B_{1}}$. If $l_{\alpha}$ is bounded by the largest number in the set $\left\{l_{\alpha[t]} \mid t<s\right\}$ (the set of all previous values of this parameter) then let the outcome be $w$.

(6) If there is an element $n$, such that $n \in \Delta_{\alpha}^{\Phi_{e}^{A_{1}}} \backslash G_{1}$ then pick the least such $n$. Suppose the least valid axiom for $n$ in $\Delta_{\alpha}$ is defined via the triple $\left\langle c, D_{\Phi}, D_{B}\right\rangle$. Enumerate $D_{B}$ back in $B_{1}$. Extract the chit $c$ from $C_{1}$. Extract from $B_{1}$ all $\Gamma_{1}$-markers for $c$ that are defined at stages $t \leq s$ when $n \notin G_{1}$. Extract $d_{\alpha}$ from $G_{2}$ and $\lambda_{2}\left(d_{\alpha}\right)$ from $A_{2}$. Initialize all lower priority strategies and let the outcome be $f$.

(7) Scan all $n \leq s$ and perform the following actions for each $n$ :

If $c(n) \uparrow$ or if $G_{1}[t] \uparrow n \neq G_{1}\left[s_{c(n)}\right] \uparrow n$ at some stage $t \in\left[s_{c(n)}, s\right]$ then let $c(n)$ be a fresh number in $C_{\alpha}$ and set $s_{c(n)}=s$ and $g_{1}(c(n))=n$. If $n \in G_{1} \backslash \Delta_{\alpha}^{\Phi_{\alpha}}$ and $c(n)<l_{\alpha}$ then find the least valid axiom $\left\langle c(n), D_{\Phi}, D_{B}\right\rangle$ in $\Theta_{\alpha}$ and enumerate the axiom $\left\langle n, D_{\Phi}\right\rangle$ in $\Delta_{\alpha}$.

Once all elements are scanned end with outcome $i$.

If $\delta[s]\left\lceil k\right.$ is an $\mathcal{R}_{e}^{2}$-strategy, the instructions are the same as above with $G_{1}, A_{1}, B_{1}, C_{1}$ swapped with $G_{2}, A_{2}, B_{2}, C_{2}$.

Case $\mathcal{Q}_{e}$ Suppose that $\delta[s]\left\lceil k\right.$ is a $\mathcal{Q}_{e}$-strategy $\beta$. Pick the first case which applies:

(1) If $s_{0}(\beta)$ is not defined then set $s_{0}(\beta)=s$. End this stage.

(2) If $s_{1}(\beta)$ is not defined then set $s_{1}(\beta)=s$. End this stage.

(3) If $G_{1}\left\lceil s_{0}(\beta)\right.$ or $G_{2}\left\lceil s_{0}(\beta)\right.$ changed at a stage $t$, such that $s_{0}(\beta)<t \leq s$, then restart $\beta$. End this stage.

(4) If there is a promise $\left\langle D_{X}, D_{G_{1}}, D_{G_{2}}\right\rangle \in P_{\beta}$, which is not currently kept: $D_{X} \subseteq X$ and $D_{G_{1}} \oplus D_{G_{2}} \nsubseteq G_{1} \oplus G_{2}$ then enumerate $D_{G_{1}}$ in $G_{1}$ and $D_{G_{2}}$ in $G_{2}$. End this stage.

(5) Let $l_{\beta}$ be the least number such that $\Psi_{\beta}^{G_{1}, G_{2}}\left(l_{\beta}\right) \neq Y\left(l_{\beta}\right)$. If there is a number $x \leq l_{\beta}$ such that $x \in \Psi^{G_{1}, G_{2}} \backslash \Xi^{X}$ then find the least valid axiom 
$\left\langle x, D_{1}, D_{2}\right\rangle \in \Psi$. Let $D_{X}=\left\{n \in X \mid\right.$ some $\Omega$-marker o(n) is in $\left.D_{1} \cup D_{2}\right\}$. Enumerate $\left\langle x, D_{X}\right\rangle$ in $\Xi$ and $\left\langle D_{X}, D_{1}, D_{2}\right\rangle$ in $P_{\beta}$. Repeat this for each such number and end this stage.

(6) If none of the above cases hold, $\beta$ has outcome $f$.

We end stage $s$ by canceling all strategies of lower priority than $\delta[s]$ and proceed to stage $s+1$.

End of construction

7.3. Verification. We start the verification with a technical lemma, which concerns promises. This lemma will then allow us to show that the constructed sets $G_{1}$ and $G_{2}$ are $\Delta_{2}^{0}$ and that the $\Lambda$-strategy succeeds.

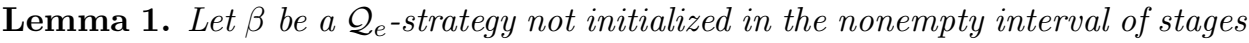
$[s, t]$. Suppose $\beta$ makes a promise $\left\langle D_{X}, D_{G_{1}}, D_{G_{2}}\right\rangle$ at stage s. If $D_{G_{1}} \oplus D_{G_{2}} \nsubseteq$ $\left(G_{1} \oplus G_{2}\right)[t]$ then there is a stage $t^{\prime} \in(s, t]$, such that $D_{X} \nsubseteq \mathbb{X}\left[t^{\prime}\right]$.

Proof. Suppose for concreteness that $o \in D_{G_{1}} \backslash G_{1}[t]$. Note that by our convention for approximating given operators we have that $o<s$. First note that $o$ cannot be a threshold of an $\mathcal{R}$-strategy as higher priority strategies do not act at stages in the interval $[s, t]$, or else $\beta$ would be initialized, and lower priority strategies are initialized at stage $s$, hence have thresholds larger than $s$. Thus $o$ is an $\Omega$-marker for some number $n$. As $o \notin G_{1}[t]$ there must be a stage $t^{\prime} \leq t$, such that $n \notin X\left[t^{\prime}\right]$. Furthermore $n \in X[s]$ or else the $\Omega$-strategy would ensure $o \notin G_{1}[s]$, contradicting $D_{G_{1}} \subseteq G_{1}[s]$. Thus $t^{\prime}>s$ and $n \in D_{X}$, thus $D_{X} \nsubseteq X\left[t^{\prime}\right]$.

Lemma 2. $G_{i}$ is $\Delta_{2}^{0}$ and $\Lambda_{i}^{A_{i}}=G_{i}$.

Proof. Fix $n$. If $n$ is a threshold then by Lemma 1 it follows that $n$ can be extracted from $G_{i}$ at most once, as $\mathcal{Q}_{e}$ strategies never need to enumerate thresholds back in $G_{i}$. If $n$ is an $\omega$-marker $n=o(x)$ then again by Lemma 1 any extraction of $n$ from $G_{i}$ corresponds to an extraction of $x$ from $X$. Hence once $X(x)$ stops changing, so will $G_{i}(n)$. As $X$ is $\Delta_{2}^{0}$, it follows that $G_{i}$ is $\Delta_{2}^{0}$.

Assume inductively that $\Lambda_{i}^{A_{i}}(m)=G_{i}(m)$ for $m<n$. First note that if $n \notin G_{i}$ then by construction when we extract $n$ from $G_{i}$, we also extract its $\lambda_{i}$-marker and hence there is no valid axiom for $n$ in $\Lambda_{i}$. Suppose that $n \in G_{i}$ then let $s_{n}$ be a stage, such that $G_{i}\left[s_{n}\right]\left\lceil n=G_{i}[t]\left\lceil n\right.\right.$ at all $t \geq s_{n}$. Then the axiom enumerated for $n$ at stage $s_{n}$ will never be invalidated again.

The goal of the proof is to show that there is a true path on the tree, consisting of strategies that are visited infinitely often and initialized finitely often. Strategies along this path will be shown to be successful. This will be done inductively and broken up into a couple of lemmas. The first one deals with $\mathcal{Q}$-strategies.

Lemma 3. Let $\beta$ be a $\mathcal{Q}_{e}$-strategy which is not initialized after stage $s_{0}$ and visited infinitely often. Then $\mathcal{Q}_{e}$ is satisfied and there is a stage $s_{\beta}$ after which $\beta$ does not end stages at which it is visited.

Proof. We may assume that strategies of higher priority than $\beta$ do not change the approximations to $G_{i}$ at stages $t>s_{0}$, otherwise $\beta$ would be initialized. After stage $s_{0}$ the strategy $\beta$ has a fixed parameter $s_{0}(\beta)$. As $G_{1}$ and $G_{2}$ are $\Delta_{2}^{0}$ by Lemma 2 there will be a least stage $s_{1} \geq s_{0}$ after which $G_{1}\left\lceil s_{0}(\beta)\right.$ and $G_{2}\left\lceil s_{0}(\beta)\right.$ do not change. At the next $\beta$-true stage after $s_{1}$ the parameter $s_{1}(\beta)$ attains its final value. After stage $s_{1}(\beta)$ Cases (1),(2) and (3) do not apply for $\beta$. 
Suppose that the sequence $\left\{l_{\beta}[t]\right\}_{t>s_{1}(\beta)}$ is unbounded. We show that $\Xi^{X}=Y$, contradicting our choice of $X$ and $Y$. Fix a natural number $x$. And suppose that $x \in \Xi^{X}$. Let $s_{x}>s_{1}(\beta)$ be the $\beta$-true stage when the least valid axiom for $x$, say $\left\langle x, D_{x}\right\rangle$, is enumerated in $\Xi$. By construction there is an axiom $\left\langle x, D_{G_{1}}, D_{G_{2}}\right\rangle \in \Psi$ and a promise $\left\langle D_{X}, D_{G_{1}}, D_{G_{2}}\right\rangle \in P$. Fix a stage $s$, such that $D_{X} \subseteq X[t]$ at all $t>s$. Then by Lemma 1 at all $\beta$-true $t>s D_{G_{1}} \subseteq G_{1}[t]$ and $D_{G_{2}} \subseteq G_{2}[t]$. Thus $x \in \Psi^{G_{1}, G_{2}}[t]$. As the the sequence $\left\{l_{\beta}[t]\right\}_{t>s_{1}(\beta)}$ is unbounded and $Y$ is $\Delta_{2}^{0}$ it follows that $x \in Y$. Suppose now that $x \in Y$. Then there are infinitely many stages $s$ such that $x \in Y[s]$ and $l_{\beta}[s]>x$. By construction at all such stages $s$, $x \in \Xi^{X}[s]$. By our choice of low approximation for $X$, it follows that $x \in \Xi^{X}$.

We have shown that there is a least number $l_{\beta}$ such that $l_{\beta}[t] \leq l_{\beta}$ at all $\beta$-true stages $t \geq s_{1}(\beta)$. It follows that there is a least number $x<l_{\beta}$ such that $Y[t](x) \neq$ $\Psi^{G_{1}, G_{2}}[t](x)$ at infinitely many stages $t$. If $x \in Y$ then $x \in Y \backslash \Psi^{G_{1}, G_{2}}$. Otherwise $x \in \Psi^{G_{1}, G_{2}}[t]$ at infinitely many stages $t$ at which $x<l_{\beta}[t]$. By construction it follows that $x \in \Xi^{X}[t]$ at infinitely many stages $t$ and hence by the lowness of $X$, $x \in \Xi^{X}$. There is a least valid axiom for $x$ in $\Xi$ for which the promise is permanently kept. It follows that $x \in \Psi^{G_{1}, G_{2}} \backslash Y$.

Furthermore for all numbers $x<l_{\beta}$ that are in $\Psi^{G_{1}, G_{2}}$ at infinitely many stages there will eventually be permanent valid axioms in the operator $\Xi$. So there will be a stage $s_{e}$ after which no new axioms are enumerated in $\Xi$ and no new promises are made. Then for every stage $t>s_{e}$ Case (5) does not apply and the set of promises does not change: $P_{\beta}[t]=P_{\beta}\left[s_{e}\right]$. Denote this final value by $P_{\beta}$. Let $n$ be larger than the maximal element of $D_{X}$ in any promise $\left\langle D_{X}, D_{G_{1}}, D_{G_{2}}\right\rangle \in P_{\beta}$. Let $s_{X}$ be the least stage after $s_{e}$, such that $X\left\lceil n\right.$ does not change after stage $s_{X}$. There are finitely many promises $\left\langle D_{X}, D_{G_{1}}, D_{G_{2}}\right\rangle \in P_{\beta}$ with $D_{X} \subseteq X$. By Lemma 1 if at stage $s \geq s_{X}$ the strategy $\beta$ keeps a promise $\left\langle D_{X}, D_{G_{1}}, D_{G_{2}}\right\rangle$ by passing through Case (4) then this promise is kept at all further stages. Thus after finitely many passes through Case (4) all of these promises are kept forever and there is a stage $s_{\beta}$, such that after stage $s_{\beta}$ the strategy $\beta$ always ends in Case (6). In this case too the strategy does not end stages prematurely.

In order to prove that the leftmost path of nodes that are visited infinitely often is the true path, we must also show that strategies along it are initialized finitely often. We we will need to consider also the actions of the $\mathcal{L}$ - and $\mathcal{R}$-strategies.

Lemma 4 . The strategy $\mathcal{L}_{e}$ satisfies its requirement and restarts $\mathcal{R}$-strategies finitely often.

Proof. Assume inductively that the statement is true for $\mathcal{L}_{e^{\prime}}$, where $e^{\prime}<e$. Let $e=\langle i, x\rangle$. There are finitely many pairs $\left(s_{0}(\alpha), \alpha\right)$, such that $\alpha$ is an $\mathcal{R}$-strategy with $s_{0}(\alpha)<e$ at some stage in the construction. For each such pair either there is a stage $t>e$ at which $\alpha$ is cancelled or there is a stage $s_{1}(\alpha)$ after which $\alpha$ is not restarted as $X\left\lceil s_{0}(\alpha)\right.$ reaches a limit and $\mathcal{L}$-strategies do not act by the inductive hypothesis. Note that the number of times $\alpha$ can change the approximation to $B_{i}$ and $C_{i}$ is bounded by the number of times it is restarted. Suppose that $s$ is a stage after all of these finitely many strategies have stopped changing the approximation to $B_{i}$ and $C_{i}$. If at all $t \geq s$ we have $x \notin \Upsilon_{i}^{B_{1}, B_{2}, C_{1}, C_{2}}[t]$ then $\mathcal{L}_{e}$ is satisfied and does not restart $\mathcal{R}$-strategies after stage $s$.

If at stage $t \geq s$ we see that $x \in \Upsilon_{i}^{B_{1}, B_{2}, C_{1}, C_{2}}[t]$ then we restart all strategies with $s_{0}(\alpha) \geq e$ and change $w_{e}$ to 1 . These strategies are activated again after stage $t$ and can only change the approximation to $B_{1}, B_{2}, C_{1}, C_{2}$ in relation to chits that 
are chosen fresh after stage $t$. Thus $x \in \Upsilon_{i}^{B_{1}, B_{2}, C_{1}, C_{2}}$ is preserved at all further stages and $\mathcal{L}_{e}$ does not restart $\mathcal{R}$-strategies after stage $t$.

Lemma 5. $B_{i}$ and $C_{i}$ are co-d.c.e

Proof. A chit $c \in C_{\alpha}$ can only be extracted once from $C_{i}$ by $\alpha$. This is because different strategies have disjoint lists of chits and when an $\mathcal{R}$-strategy $\alpha$ is initialized, $C_{\alpha}$ is restarted and becomes defined to contain only fresh numbers. Similarly, a $\Gamma_{i}$-marker $\gamma_{i}(c)$ can only be extracted once from $B_{i}$ by the strategy $\alpha$, such that $c \in C_{\alpha}$.

Lemma 6. Let $\alpha$ be an $\mathcal{R}_{e}^{i}$-strategy which is not initialized after stage $s_{0}$ and visited infinitely often. Then $\mathcal{R}_{e}^{i}$ is satisfied and there is a stage $s_{\alpha}$ after which $\alpha$ does end stages at which it is visited. If $\alpha$ extracts a chit $c$ from $C_{i}$ after stage $s_{0}$ then $c \notin \Gamma_{i}^{B_{i}, G_{i}}$.

Proof. Suppose for concreteness $i=1$. After stage $s_{0}$ the parameter $s_{0}(\alpha)$ does not change and higher priority $\mathcal{R}$ - and $\mathcal{Q}$-strategies do not make any further changes to any of the global parameters: $A_{i}, C_{i}, B_{i}$, and $G_{i}$. As $X$ is $\Delta_{2}^{0}$ and by Lemma $4 \mathcal{L}$-strategies with index $i<s_{0}(\alpha)$ act finitely many times, there will be a least stage $s_{1} \geq s_{0}$ after which $\alpha$ does not get restarted. At the next $\alpha$-true stage after $s_{1}$ the parameters $s_{1}(\alpha), d_{\alpha}$ and $C_{\alpha}$ will attain their final value. The final value of $d_{\alpha}$ is $\omega\left(s_{0}(\alpha)\right)\left[s_{1}\right]+1$. As $X\left\lceil s_{0}\right.$ does not change at any further stage it follows that $G_{1}\left\lceil d_{\alpha}\right.$ does not change at any further stage. After stage $s_{1}(\alpha)$ Cases (1), (2) and (3) do not apply for $\alpha$.

Suppose that there is a stage $s$ at which Case (6) applies for $\alpha$. There is a number $n \in \Delta_{\alpha}^{\Phi_{e}^{A_{1}}} \backslash G_{1}[s]$. At stage $s$ the threshold $d_{\alpha}$ is extracted from $G_{2}$ and all lower priority strategies are initialized. It follows from the proof of Lemma 1 that $d_{\alpha}$ remains out of $G_{2}[t]$ at all further stages $t$, hence $G_{1}\lceil s$ does not change at any further stage, in particular $n \notin G_{1}$. From this we get that $A_{1}\lceil s$ and hence $\Phi_{e}^{A_{1}}\left\lceil s \subseteq \Phi_{e}^{A_{1}}\right.$. Suppose that the valid axiom for $n$ in $\Delta_{\alpha}$ was defined via the triple $\left\langle c, D_{\Phi}, D_{B}\right\rangle$ at stage $t<s$. Then $c$ was assigned to $n$ at stage $s_{c}<t$. At stage $s_{c}$ the chit $c$ was selected as a fresh number, i.e. no axiom for $c$ enumerated in $\Gamma_{1}$ before stage $s_{c}+1$. The marker $g_{1}(c)$ was set to equal $n$. Thus all axioms for $\Gamma_{1}$ defined at stages at which $n \in G_{1}$ are invalid. At stage $s$ the strategy extracts also all $\Gamma_{1}$-markers defined at stages at which $n \notin G_{1}$. These markers are defined after stage $t$ and hence are all larger than $\max D_{B}$.

At stage $s$ all lower priority strategies are initialized so that $B_{1}[s]\left\lceil s \subseteq B_{1}\right.$ at all further stages. Thus $c \in \Theta_{e}^{\Phi_{e}^{A_{1}}, B_{1}} \backslash C_{1}$ and the requirement is satisfied. Furthermore we have shown that $c \notin \Gamma_{1}^{B_{1}, G_{1}}$.

If Case (6) never applies for $\alpha$ after stage $s_{1}(\alpha)$ then neither does Case (4). Hence $\alpha$ does not end prematurely any true stage $t>s_{1}(\alpha)$ and does not extract any numbers from $B_{1}$ or $C_{1}$.

Suppose that there is a stage $s_{5}$, such that at all $\alpha$-true stages $t>s_{5}$ Case (5) applies for $\alpha$. It follows that there is a number $x$ such that at infinitely many stages $t>s_{5}$ there $x \leq l_{\alpha}[t]$ and $x \in C_{1}[t] \backslash \Theta_{e}^{\Phi_{e}^{A_{1}}, B_{1}}[t]$. By Lemma 5 the set $C_{1}$ is co-d.c.e, hence $\Theta_{e}^{\Phi_{e}^{A_{1}}, B_{1}} \neq C_{1}$ and $\mathcal{R}_{e}^{1}$ is satisfied.

Finally suppose that at infinitely many $\alpha$-true stages Case (7) applies. Suppose that $\Theta_{e}^{\Phi_{e}^{A_{1}}, B_{1}}=C_{1}$. We will show that $\Delta_{\alpha}^{\Phi_{e}^{A_{1}}}=G_{1}$. Fix a natural number $n$. If 
$n \in G_{1}$ then by Lemma 2 we know that $G_{1}\lceil n$ will eventually stop changing and $n$ will be assigned final chit $c(n) \in C_{1}$. As $\Theta_{e}^{\Phi_{e}^{A_{1}}, B_{1}}=C_{1}$ there will be a least valid axiom for $c(n)$ in $\Theta_{e}$ that is valid at all large enough stages. So eventually a valid axiom for $n$ will be enumerated in $\Delta_{\alpha}$. If $n \notin G_{1}[s]$ at an $\alpha$-true stage $s$ then $n \notin \Delta_{\alpha}^{\Phi_{e}^{A_{1}}}[s]$ or else Case (6) would apply for $n$.

From Lemma 3 and Lemma 6 we get immediately the True path lemma.

Corollary 2. There is an infinite path $f$ in the tree, such that for every $n, f\lceil n$ is visited infinitely often and initialized only finitely often. We call it the true path. All $\mathcal{R}$ and $\mathcal{Q}$ requirements are satisfied.

We complete the verification with two lemmas, showing that the strategies $\Gamma_{1}$, $\Gamma_{2}$ and $\Omega$ succeed.

Lemma 7. $\Gamma_{i}^{B_{i}, G_{i}}=C_{i}$

Proof. Suppose that $\Gamma_{i}^{B_{i}, G_{i}}(m)=C(m)$ for $m<c$ and let $s_{c}$ be a stage, such that $C_{i}\left\lceil c\right.$ does not change after stage $s_{c}$. If $c \in C_{i}$ then $B_{i}\left\lceil\gamma_{i}(c)\right.$ does not change after stage $s_{c}$. Hence once the approximation to $G_{i}\left\lceil g_{i}(c)\right.$ has also settled, there will be an axiom for $c$ in $\Gamma_{i}$ which will never be invalidated. Suppose that $c \notin C_{i}$. Then $c$ is a chit for an element $n$ used by an $\mathcal{R}^{i}$-strategy $\alpha$ in an attack at stage $s$ and $\alpha$ is not initialized after stage $s$. It follows from the Lemma 6 that $c \notin \Gamma_{i}^{B_{i}, G_{i}}$.

Lemma 8. $\Omega^{G_{1}, G_{2}}(n)=X(n)$.

Proof. At every stage $s$ the $\Omega$-strategy ensures that for every $x<s, \Omega^{G_{1}, G_{2}}(x)[s]=$ $X(x)[s]$. Thus if $x \notin X$ then $x \notin \Omega^{G_{1}, G_{2}}$. If $x \in X$ then let $s_{x}>x$ be a stage, such that $X\left\lceil x\right.$ does not change at stages larger than $s_{x}$. Consider the first $\mathcal{R}$-strategy along the true path with $s_{0}=s_{0}(\alpha)>s_{x}$. Let $s_{1}$ be the final value of the parameter $s_{1}(\alpha)$. It follows from Lemma 6 that $\left(G_{1} \oplus G_{2}\right)\left[s_{1}\right]\left\lceil\omega\left(s_{0}\right)\right.$ does not change after stage $s_{1}$. Hence the axiom enumerated at stage $s_{1}$ for $x$ in $\Omega$ is valid at all further stages.

\section{Determining the LOW CO-D.C.e. EnUmeration DEgRees From the the \\ CO-C.E. DEGREES}

In this section we explain how to modify the construction from the previous section to show that Theorem 10 is true. Let $X$ be a low co-d.c.e. set and $Y$ be a $\Delta_{2}^{0}$ set, such that $Y \not_{e} X$. We wish to construct $\Pi_{1}^{0}$ sets $A_{i}, B_{i}, C_{i}$, co-d.c.e. sets $G_{i}$ for $i=1,2$ to satisfy the same list of requirements, excluding the lowness requirements. For the requirements $\Lambda_{i}$, stating that $G_{i}=\Lambda_{i}^{A_{i}}$, and $\Gamma_{i}$, stating that $C_{i}=\Gamma_{i}^{B_{i}, G_{i}}$, we use the exact same strategies as in the previous section. Similarly we treat the list of requirements $\left\{\mathcal{Q}_{e}\right\}_{e<\omega}$, where $\mathcal{Q}_{e}$ states that $\Psi_{e}^{G_{1}, G_{2}} \neq Y$, exactly the same. We will only modify the strategies for the $\Omega$-requirement, stating that $X=\Omega^{G_{1}, G_{2}}$ and for the list of $\mathcal{R}$-requirements, where $\mathcal{R}_{e}^{i}$ states that if $\Theta_{e}^{\Phi_{e}^{A_{i}}, B_{i}}=C_{i}$ then there is an enumeration operator $\Delta_{e}$, such that $G_{i}=\Delta_{e}^{\Phi_{e}^{A_{i}}}$.

\subsection{Description of the modified strategies and the construction.}

The $\Omega$-strategy. In this case as well $\Omega$ will define an $\Omega$-marker $\omega(n)$ for every natural number $n$. We will distinguish between the current $\Omega$-marker $\omega(x)$ and 
previously defined $\Omega$-markers $o(x)$. The marker $\omega(x)$ will always be chosen as a fresh number, so that $\omega(x) \in G_{1} \cap G_{2}$. Every axiom enumerated in $\Omega$ for $x$, say $\left\langle x, D_{1}, D_{2}\right\rangle$ will include some marker $o(x)$ in both $D_{1}$ and $D_{2}$. Thus extracting $o(x)$ from either oracle set will invalidate the axiom. The modification is that now $\mathcal{R}_{e}$-strategies will make the choice whether $o(x)$ will be extracted from $G_{1}$ or from $G_{2}$. For this reason $o(x)$ will come with a flag $i(o(x))$ which has values 1 or 2 . Initially $i(o(x))=1$. At stage $s$ the strategy makes sure that $\Omega^{G_{1}, G_{2}}[s]=X[s]$ by enumerating axioms for numbers in $X[s]$ and extracting numbers from $G_{1}$ or $G_{2}$ to invalidate axioms for numbers not in $X[s]$. If $o(x)$ needs to be extracted from $G_{1}$ or $G_{2}$ then it will be extracted from $G_{i(o(x))}$.

We replace Step $I$ in the construction by the following:

Step I: $\Omega$ : Scan all $n<s$.

(1) If $x \in X \backslash \Omega^{G_{1}, G_{2}}$ then let $\omega(x)$ be a fresh number and enumerate in $\Omega$ the axiom $\left\langle x, G_{1}\left\lceil\omega(x), G_{2}\lceil\omega(x)\rangle\right.\right.$ (the axiom is defined with marker $\omega(x)$ ). Set $i(\omega(x))=1$.

(2) If $x \in \Omega^{G_{1}, G_{2}} \backslash X$ then for every valid axiom for $x$ in $\Omega$ defined with marker $o(x)$ : extract $o(x)$ from $G_{i(o(x))}$ and $\lambda_{i(o(x))}(o(x))$ from $A_{i(o(x))}$.

Steps II and III remain the same and Step IV is deleted. To complete the construction we first explain how the $\mathcal{R}$-strategies must be modified.

The $\mathcal{R}$-strategies. We will not be able to use the same design for the $\mathcal{R}$-strategies as in the proof of Theorem 9 . The reason is that the sets $B_{i}$ and $C_{i}$ have to be $\Pi_{1}^{0}$. Thus once a number is extracted from either set, it must remain extracted at all further stages. This creates a potential conflict between $\mathcal{R}$-strategies of the same kind. A lower priority $\mathcal{R}^{i}$-strategy $\alpha^{\prime}$ might need to diagonalize and extract some number $b$ from $B_{i}$ to keep $\Gamma_{i}$ correct. A higher priority $\mathcal{R}^{i}$-strategy $\alpha$ might be counting on $b \in B_{i}$. It has used a triple $\left\langle c, D_{\Phi}, D_{B}\right\rangle$ with $b \in B_{i}$ to define an axiom for a number $n$ in $\Delta_{\alpha}$. If $n$ leaves the approximation to $G_{i}$ and $D_{b} \nsubseteq B_{i}$, there is no way to correct the operator $\Delta_{\alpha}$. To resolve this conflict we will design the $\mathcal{R}$-strategies so that they are more careful about preserving the work of higher priority strategies. We introduce three modifications.

Suppose that $\alpha$ is working towards satisfying the requirement $\mathcal{R}_{e}^{1}$. It will as in the previous proof first define its parameter $s_{0}(\alpha)$ and wait until $X \uparrow s_{0}(\alpha)$ stops changing. It will be restarted every time a change is observed. Then at stage $s_{1}(\alpha)$ it will define its threshold $d_{\alpha}$. But it will not yet select its list of chits. Before the strategy goes on to satisfying its own requirement - diagonalizing $\Theta^{\Phi^{A_{1}}, B_{1}}$ against $C_{1}$ or constructing an operator $\Delta_{\alpha}$, such that $\Delta_{\alpha}^{\Phi^{A_{1}}}=G_{1}$, it first works towards ensuring that its attempts at diagonalization will not injure higher priority $\mathcal{R}_{1}$ strategies that are also constructing their own operators. The strategy $\alpha$ must ensure that all higher priority strategies have defined their final axioms for numbers $n \leq d_{\alpha}$. To do this the strategy will wait until all of these strategies have enumerated in their operators axioms for every $m \leq d_{\alpha}$, such that $m \in G_{1}$. For every higher priority $\mathcal{R}_{1}$-strategy $\alpha^{\prime}$, such that $\alpha$ believes that $\alpha^{\prime}$ is building an enumeration operator $\Delta_{\alpha^{\prime}}$, i.e. such that $\alpha^{\prime \wedge} i \preceq \alpha$, the strategy $\alpha$ will set the flag $i(n)$ to 2 for all numbers $n>d_{\alpha}$ necessary to preserve some axiom that $\alpha^{\prime}$ has defined. Note this is a finite process as $G_{1}\left\lceil d_{\alpha}\right.$ does not change at any further stage and once an axiom is preserved it will remain valid at all further stages, as lower priority $\mathcal{R}^{2}$-strategies will be initialized at these stages. We denote by $s_{2}(\alpha)$ the 
stage when $\alpha$ has completed this task. At this stage the strategy $\alpha$ finally selects its list of chits $C_{\alpha}$, containing numbers larger than $s_{2}(\alpha)$.

Next $\alpha$ examines the largest number $l_{\alpha}$ such that $C_{1} \uparrow l_{\alpha} \subseteq \Theta^{\Phi^{A_{1}}, B_{1}}$, to determine whether or not the stage is expansionary and has outcome $w$ if it is not. If the stage is expansionary $\alpha$ proceeds to check if $\Delta_{\alpha}$ is correct and to add more axioms for it if necessary.

Here as well we use chits to define axioms in $\Delta_{\alpha}$. Every natural number $n \in G_{1}$ is assigned a chit $c(n) \in C_{\alpha}$. When this chit is assigned it is a fresh number, the strategy $\Gamma_{1}$ has not yet defined its $g_{1}$-marker. We set $g_{1}(c(n))$ to $n$. We change the chit for $n$ every time $G_{1}\left\lceil n\right.$ changes, so that all axioms for $c(n)$ in $\Gamma_{i}$ assume that $n \in G_{1}$. If there is no valid axiom for $n$ in $\Delta_{\alpha}$ and there is a valid axiom $\left\langle c(n), D_{\Phi}, D_{B}\right\rangle$ for $c(n)$ in $\Theta$ we define a new axiom for $n$ in $\Delta_{\alpha}$. Here is where we introduce the second modification: the axiom for $n$ will include all currently valid axioms for numbers $m<n$, such that $m \in G_{1}$. This will ensure that if $\alpha$ later on needs to attack with $n$ then all numbers $m<n$ that were in $G_{1}$ when we defined the axioms for $n$ are also in $G_{1}$ at the stage of the attack.

Finally if $\alpha$ sees a number $n$, such that $n \in \Delta_{\alpha}^{\Phi^{A_{1}}} \backslash G_{1}(n)$ and the valid axiom for $n$ is defined using the triple $\left\langle c(n), D_{\Phi}, D_{B}\right\rangle$, then we extract $c(n)$ from $C_{1}$ and extract from $B_{1}$ the least markers $\gamma(c(n))$ used in an axiom which assumes $n \notin G_{1}$. We extract $d_{\alpha}$ from $G_{2}$ and dump all $m>n$ in $G_{1}$. This is the third modification.

Let us reevaluate the possible conflict described above with the newly designed strategy. It will follow from the construction that $G_{1}$ is co-d.c.e. Thus a number can be extracted only once from $G_{1}$. If $\alpha^{\prime}$ is a lower priority strategy which attacks with $n$ at stage $s$ then by the first modification it will be a attacking relative to a chit is defined after all axioms for $m<d_{\alpha^{\prime}}$ in $\Delta_{\alpha}$ are already defined, and hence the change in $B_{1}$ is not on a number used in any axiom for $m<d_{\alpha^{\prime}}$. By the third modification all $m>n$ are dumped in $G_{1}$ at the stage of the attack and will never again leave $G_{1}$, so $\alpha$ will never have to attack relative to them. Similarly, any number in the interval $\left[d_{\alpha^{\prime}}, n\right]$ that is in $G_{1}$ at the stage of the attack will remain in $G_{1}$ at all further stages due to the extraction of the threshold $d_{\alpha^{\prime}}$ by $\alpha^{\prime}$. Any number in the interval $\left[d_{\alpha^{\prime}}, n\right)$ that is not in $G_{1}$ at the stage of the attack is already out when $\alpha^{\prime}$ defined the axiom of attack by the second modification, and $\alpha^{\prime}$ extracts numbers defined after this stage. This leaves $n$ and the possibility that $\alpha^{\prime}$ extracts a number out of $B_{1}$, that invalidates the axiom that is used by $\alpha$ for $n$ in the definition of $\Delta_{\alpha}$. But as $n$ is extracted only once from $G_{1}$ it follows that $\alpha^{\prime}$ preserves any number seen in any axiom while $n \in G_{1}$. Thus the conflict is resolved.

We modify Case $\mathcal{R}_{e}^{i}$ from Step V of the construction as follows:

Case $\mathcal{R}_{e}^{i}$ Suppose that $\delta[s] \uparrow k$ is an $\mathcal{R}_{e}^{1}$-strategy $\alpha$. The strategy has three additional parameters: $s_{2}(\alpha)$, the stage when $\alpha$ can start its work; $m_{\alpha}$, the largest number scanned during a visit and $R_{\alpha}$, the largest number from the set $A_{1}$ used in an axiom for $\Delta_{\alpha}$. Let $s^{-}$be the previous stage at which $\alpha$ was visited and $o^{-}$be the outcome that $\alpha$ had at stage $s^{-}$. (If $\alpha$ has never been or has been initialized since stage $s^{-}$then let and $o^{-}=w$ ). Pick the first case which applies to $\alpha$ :

(1) If $s_{0}(\alpha)$ is not defined then set $s_{0}(\alpha)=s$. End this stage.

(2) If $s_{1}(\alpha)$ is not defined then set $s_{1}(\alpha)=s$ and $d_{\alpha}=\omega\left(s_{0}(\alpha)\right)+1$. End this stage. 
(3) If $s_{1}(\alpha)$ is defined, but $X\left\lceil s_{0}(\alpha)\right.$ changed at a stage $t$, such that $s_{1}(\alpha)<$ $t \leq s$, then restart $\alpha$. End this stage.

(4) If $\alpha^{\prime \wedge} i \preceq \alpha$ and $m_{\alpha^{\prime}} \leq d_{\alpha}+1$ or a new axiom for an element $m<d_{\alpha}$ was enumerated in $\Delta_{\alpha^{\prime}}$ since stage $s^{-}$then for every $n$, such that $d_{\alpha}<n$ and $\lambda_{1}(n) \leq R_{\alpha^{\prime}}$, set $i(n)=2$. Make $s_{2}(\alpha), C_{\alpha}$ and $\Delta_{\alpha}$ undefined. End this stage.

(5) If $s_{2}(\alpha)$ is not defined then set $s_{2}(\alpha)=s$. Set $C_{\alpha}=\left\{\langle\hat{\alpha}, n\rangle \mid n>s_{2}(\alpha)\right\}$ where $\hat{\alpha}$ is the code of $\alpha$ in some fixed computable coding of all finite binary strings. End this stage.

(6) If the outcome at stage $s^{-}$was $f$ then let the outcome be $f$.

(7) If the current stage is not expansionary then let the outcome be $w$.

(8) If there is an element $n$, such that $n \in \Delta_{\alpha}^{\Phi_{e}^{A_{1}}} \backslash G_{1}$, then pick the least such $n$. Suppose the least valid axiom for $n$ in $\Delta_{\alpha}$ is defined via the triple $\left\langle c, D_{\Phi}, D_{B}\right\rangle$ and this is a valid axiom in $\Theta_{e}$. Extract the chit $c$ from $C_{1}$, extract from $B_{1}$ the least marker $\gamma_{1}(c)$ which is used in an axiom that assumes $n \notin G_{1}$. Extract $d_{\alpha}$ from $G_{2}$ and $\lambda_{2}\left(d_{\alpha}\right)$ from $A_{2}$. Enumerate all numbers $m \in(n, s]$ in $G_{1}$. Initialize all lower priority strategies and let the outcome be $f$.

(9) Scan all $n \leq s$, such that $n \in G_{1}$, and perform the following actions:

If $c(n) \uparrow$ or if $G_{1}\left\lceil n[t] \neq G_{1} \uparrow n\left[s_{c(n)}\right]\right.$ at some stage $t \geq s_{c(n)}$ then let $c(n)$ be a fresh number in $C_{\alpha}$ and set $s_{c(n)}=s$ and $g_{1}(c(n))=n$. If $c(n)$ is greater than the length of agreement between $\Theta_{e}^{\Phi_{e}^{A_{1}}, B_{1}}$ and $C_{1}$ then stop the scan. Otherwise if $n \notin \Delta_{\alpha}^{\Phi_{\alpha}}$ then find the least valid axiom $\left\langle c(n), D_{\Phi}, D_{B}\right\rangle$ in $\Theta_{e}$. Let $D_{n}$ be the union of the currently valid axioms for $m<n$, such that $m \in G_{1}$, and enumerate the axiom $\left\langle n, D_{n} \cup D_{\Phi}\right\rangle$ in $\Delta_{\alpha}$.

Once the scan has been completed (or stopped) define $m_{\alpha}$ as the largest scanned element for which there is a valid axiom in $\Delta_{\alpha}$. We set $R_{\alpha}$ to be the largest element that is in a $\Phi_{e}$-axiom for an element in a $\Delta_{\alpha}$ axiom and end with outcome $i$.

If $\delta[s]\left\lceil k\right.$ is an $\mathcal{R}_{e}^{2}$-strategy, the instructions are the same as above with $G_{1}, A_{1}, B_{1}, C_{1}$ swapped with $G_{2}, A_{2}, B_{2}, C_{2}$.

8.2. Verification. We start with a technical lemma that deals with the modified $\mathcal{R}$-strategies and serves as an important tool for unravelling the construction.

Lemma 9. Let $\alpha$ be an $\mathcal{R}_{e}^{1}$-strategy which extracts its threshold $d_{\alpha}$ from $G_{2}$ at stage $s$ to perform a to diagonalization relative to a number $n$. Suppose the valid axiom for $n$ in $\Delta_{\alpha}$ is defined via the triple $\left\langle c(n), D_{\Phi}, D_{B}\right\rangle$ at stage $s^{\prime}<s$. Then at all stages $t>s$ :

(1) There is threshold $d_{\alpha^{\prime}} \leq d_{\alpha}$ for a strategy $\alpha^{\prime} \leq \alpha$, such that

$$
d_{\alpha^{\prime}} \in\left(G_{1} \cap G_{2}\right)[s-1] \text { and } d_{\alpha^{\prime}} \notin\left(G_{1} \cup G_{2}\right)[t] .
$$

(2) If $G_{1}\left\lceil n\left[s^{\prime}\right] \subseteq G_{1}[t]\right.$ then $\left(B_{1}\left\lceil\gamma_{1}(c(n))\right)\left[s^{\prime}\right] \nsubseteq B_{1}[t]\right.$

(3) All numbers $m$ in the interval $\left[d_{\alpha}, s\right]$, such that $m \in G_{1}[s]$ are in $G_{1}[t]$.

The symmetric statement with 1 and 2 swapped is also true.

Proof. Suppose that the statement is true for all $\alpha^{\prime}$ of higher priority than $\alpha$. The strategy $\alpha$ selects its threshold $d_{\alpha}$ at stage $s_{1}(\alpha)<s$ as $\omega\left(s_{0}(\alpha)\right)+1$ and $\alpha$ is 
not initialized between stages $s_{0}(\alpha)$ and $s$. At stage $s$ it extracts $d_{\alpha}$ from $G_{2}$. First we show that a $\mathcal{Q}$-strategy $\beta$ does not enumerate $d_{\alpha}$ back in $G_{2}$. Towards a contradiction suppose that a $\mathcal{Q}$-strategy $\beta$ has a promise $\left\langle D_{X}, D_{G_{1}}, D_{G_{2}}\right\rangle$ with $d_{\alpha} \in D_{G_{2}}$. This promise must have been made before stage $s$ by $\beta$ and $\beta$ must have higher priority than $\alpha$. Lower priority strategies are initialized at stage $s$ and when a $\mathcal{Q}$-strategy is initialized it moves the value of its parameter $s_{0}(\beta)$ and restarts every time a change in $G_{i} \uparrow s_{0}(\beta)$ for $i=1,2$ is observed. But in this case, as $\alpha$ is not initialized between stages $s_{0}(\alpha)$ and $s$, it follows that $\beta$ made this promise before stage $s_{0}(\alpha)$, hence $\max D_{G_{2}}<s_{0}(\alpha)<d_{\alpha}$. So if $d_{\alpha} \in G_{2}[t]$ for $t>s$ then an $\mathcal{R}^{2}$-strategy $\alpha^{\prime}$ must be responsible for this. Again strategies that select their thresholds after stage $s$ have thresholds of value greater than $d_{\alpha}$ and cannot enumerate $d_{\alpha}$ in $G_{2}$. This rules out lower priority strategies. It follows that $\alpha^{\prime}$ is of higher priority than $\alpha$ and at stage $t$ it extracts from $G_{1}$ its own threshold $d_{\alpha^{\prime}}<d_{\alpha}$. Thus $d_{\alpha^{\prime}} \in G_{1}[s-1] \backslash G_{1}[t]$. By induction the statement follows.

Now similarly suppose that $n$ is enumerated back in $G_{1}$ at some stage $t \geq s$. The same argument as the one above shows that $n$ is not enumerated back in $G_{1}$ by a $\mathcal{Q}$-strategy $\beta$, or by a lower priority $\mathcal{R}$-strategy. Thus this must be done by a higher priority $\mathcal{R}^{1}$-strategy $\alpha^{\prime}$ which attacked at stage $t$ with a number $m<n$. Pick the least number $m$, such that some $\mathcal{R}$-strategy $\alpha^{\prime}$ of higher priority attacks at some stage $t>s$ with $m$. Then $m \notin G_{1}\left[t^{\prime}\right]$ at all $t^{\prime} \geq t$. If $m \in G_{1}\left[s^{\prime}\right]$ then this proves the statement. If $m \notin G_{1}\left[s^{\prime}\right]$ then the chit $c^{\prime}(m)$ for $m$ at $\alpha^{\prime}$ was selected while $m \in G_{1}$ hence before stage $s^{\prime}$, and even before the chit $c(n)$ was assigned to $n$ at $\alpha$. At stage $s^{\prime}$ we have that $m \notin G_{1}\left[s^{\prime}\right]$ hence the least marker for $c^{\prime}(m)$ that assumes $m \notin G_{1}$ is already defined, it is in $B_{1}\left[s^{\prime}\right]$ and it is smaller than $\gamma_{1}(c(n))\left[s^{\prime}\right]$. As $\alpha^{\prime}$ extracts this marker from $B_{1}$ at stage $t$ it follows that $\left(B_{1}\left\lceil\gamma_{1}(c(n))\right)\left[s^{\prime}\right] \nsubseteq s_{1}[t]\right.$.

Finally let $m \in\left[d_{\alpha}, s\right]$ and $m \in G_{1}[s]$. As higher priority strategies have thresholds smaller than $m$ at stage $s$ and lower priority strategies are initialized at stage $s$ it follows that $m$ cannot be extracted by an $\mathcal{R}$-strategy. If $m$ is an $\omega$-marker used in some axiom $\left\langle x, D_{1}, D_{2}\right\rangle$ in $\Omega$ then this axiom is defined in the time period $\left(s_{0}(\alpha), s\right]$ and hence must have $\left(G_{1}\left\lceil d_{\alpha} \oplus G_{2}\left\lceil d_{\alpha}\right)[s-1] \subseteq D_{1} \oplus D_{2}\right.\right.$. By the first statement in this lemma it follows that this axiom is invalid at all further stages and so the strategy $\Omega$ will never extract it from $G_{1}$.

Now we can establish that $G_{1}$ and $G_{2}$ are co-d.c.e. sets. As in the previous section this lets us conclude that the $\Lambda$-strategies are successful.

Lemma 10. $G_{1}$ and $G_{2}$ are co-d.c.e. and $\Lambda_{i}^{A_{i}}=G_{i}$.

Proof. We show that $G_{1}$ is co-d.c.e. Let $n$ be a natural number. Then $n$ is extracted from $G_{1}$ for two reasons: either it is a threshold for some $\mathcal{R}^{2}$-strategy or else it is an $\Omega$-marker for some natural number $x$. If $n$ is a threshold then it is the threshold of a unique strategy $\alpha$, it is not an $\Omega$-marker and can only be extracted from $G_{1}$ once by $\alpha$. This is ensured by the way we select thresholds: relative to the $\Omega$-marker of the first stage after initialization of $\alpha$. At this stage $\alpha$ initializes lower priority strategies and reserves this stage for its own use.

If $n$ is an $\omega$-marker $n=o(x)$ then it can be extracted only by the strategy $\Omega$ at stages $s$, such that $x \notin X[s]$. Once it is extracted, it can be enumerated back by a $\mathcal{Q}$-strategy at a further stage $t \geq s$, only if there is a promise $\left\langle D_{X}, D_{G_{1}}, D_{G_{2}}\right\rangle$, such that $x \in D_{X}$ and $n \in D_{1}$ and $D_{x} \subseteq X[t]$. But as $X$ is co-d.c.e. it follows that $x \in X\left[t^{\prime}\right]$ at all further stages $t^{\prime} \geq t$ and hence $\Omega$ will not extract $n$ a second time. It can also be enumerated back by an $\mathcal{R}^{1}$-strategy $\alpha$ only when $\alpha$ attacks with a 
number $m<n$ at stage $t>n$. But then by Lemma 9 we have that $n \in G_{1}$ at all further stages. It follows that $G_{1}$ is co-d.c.e.

A similar proof shows that $G_{2}$ is co-d.c.e. The proof that $\Lambda_{i}^{A_{i}}=G_{i}$ is can now be implemented as in Lemma 2.

We next show that the $\Gamma_{i}$ strategies succeed.

Lemma 11. $\Gamma_{i}^{B_{i}, G_{i}}=C_{i}$

Proof. Suppose that $\Gamma_{i}^{B_{i}, G_{i}}(m)=C_{i}(m)$ for $m<c$ and let $s_{n}$ be a stage, such that $C_{i}\left\lceil c\right.$ does not change after stage $s_{c}$. If $c \in C_{i}$ then $B_{i}\left\lceil\gamma_{i}(c)\right.$ does not change after stage $s_{c}$. Hence once the approximation to $G_{i}\left\lceil g_{i}(c)\right.$ has also settled, there will be an axiom for $c$ in $\Gamma_{i}$ which will never be invalidated. Suppose that $c \notin C_{i}$. Then $c$ is a chit for an element $n$ used by an $\mathcal{R}^{i}$-strategy $\alpha$ in an attack at stage $s$. Then $g_{i}(c)=n$ and at stage $s \alpha$ extracted from $B_{i}$ the least marker $\gamma_{i}(c)$ used in an axiom that assumes $n \notin G_{i}$. The structure of the axioms in $\Gamma_{i}$ ensures that this will invalidate all later axioms for $c$ defined until stage $s$. By Step (2) of Lemma 9 the axioms that assume $n \in G_{i}$ are also invalid at all further stages.

Lemma 12. Let $\alpha$ be an $\mathcal{R}_{e}^{i}$-strategy which is not initialized after stage $s_{0}$ and visited infinitely often. Then $\mathcal{R}_{e}^{i}$ is satisfied and there is a stage $s_{\alpha}$ after which $\alpha$ does end stages at which it is visited.

Proof. For concreteness let $i=1$. After stage $s_{0}$ the parameter $s_{0}(\alpha)$ does not change and higher priority $\mathcal{R}$ - and $\mathcal{Q}$-strategies do not make any further changes to any of the global parameters: $A_{i}, C_{i}, B_{i}$, and $G_{i}$. As $X$ is co-d.c.e. there will be a least stage $s_{1} \geq s_{0}$ after which $X\left\lceil s_{0}(\alpha)\right.$ does not change. At the next $\alpha$-true stage after $s_{1}$ the parameters $s_{1}(\alpha)$ and $d_{\alpha}$ attain their final value. The final value of $d_{\alpha}$ is $\omega\left(s_{0}(\alpha)\right)\left[s_{1}\right]+1$. As $X\left\lceil s_{0}\right.$ does not change at any further stage it follows that $G_{1}\left\lceil d_{\alpha}\right.$ does not change at any further stage. After stage $s_{1}(\alpha)$ Cases (1), (2) and (3) do not apply for $\alpha$.

Suppose that $\alpha^{\prime}$ is a higher priority $\mathcal{R}_{e^{\prime}}^{1}$-strategy, such that $\alpha^{\prime \wedge} i \subseteq \alpha$. As $\alpha$ is visited at infinitely many stages, it follows that $\alpha^{\prime}$ has infinitely many expansionary stages. Fix $m \leq d_{\alpha}$. As $G_{1}\left\lceil m\right.$ does not change after stage $s_{1}(\alpha)$, the $\alpha^{\prime}$-chit for $m, c^{\prime}(m)$, does not change after stage $s_{1}(\alpha)$. Hence once the length of inclusion examined by $\alpha^{\prime}$ exceeds $c^{\prime}(n)$, the strategy $\alpha^{\prime}$ enumerates a valid axiom for $n$ in $\Delta_{\alpha^{\prime}}$. At the first stage when $\alpha$ is visited after that it sets the flag $i(x)=2$ for every number $x \geq d_{\alpha}$, such that a change in $G_{1}(x)$ can cause a change in $A_{1}\left\lceil R_{\alpha^{\prime}}\right.$. As higher priority strategies do not act and lower priority strategies are initialized at this stage, no one can change the flag back to 1 . Finally as by assumption $X\left\lceil s_{0}\right.$ and hence $G_{1} \uparrow d_{\alpha}$ do not change after stage $s_{1}(\alpha)$, it follows that $A_{1} \uparrow R_{\alpha^{\prime}}$ is preserved at all further stages and hence the axiom for $m$ in $\Delta_{\alpha^{\prime}}$ remains valid at all further stages. It follows from this analysis that there is a stage $s_{2}$, such that at all stages $t \geq s_{2}$ all higher priority strategies $\alpha^{\prime}$ have $m_{\alpha^{\prime}}>d_{\alpha}$ and do not enumerate more axioms for elements $m<d_{\alpha}$. At the first $\alpha$-true stage after stage $s_{2}$ the final values of the parameters $s_{2}(\alpha)$ and $C_{\alpha}$ are defined. Cases (4) and (5) do not apply to $\alpha$ at any further stage.

Suppose that Case (6) applies for $\alpha$ at stage a least stage $s_{6}$. Then at all further stages $t \geq s_{6}$ the strategy $\alpha$ ends with outcome $f$ and does not initialize lower priority strategies. Consider the $\alpha$-true stage before $s_{6}$, call it $s$. At stage $s$ Case (8) applies for $\alpha$ and $d_{\alpha}$ was extracted from $G_{2}$ and all lower priority strategies are initialized. By Lemma 9 as higher priority strategies do not act we know that $G_{1}\lceil s$ 
and hence $A_{1}\lceil s$ do not change at further stages and hence no number can leave the approximation to $\Phi_{e}^{A_{1}}\left\lceil s\right.$ at further stages. There is a chit $c \in C_{\alpha}[s]$, such that $c \in \Theta_{e}^{\Phi_{e}^{A_{1}}, B_{1}}(c)[s]$ via the valid axiom $\left\langle c, D_{\Phi}, D_{B}\right\rangle$. We extract $c$ from $C_{1}$ and extract from $B_{1}$ only $\gamma_{1}(c)$-markers that assume that $n \notin G_{1}$, hence elements larger than $\max D_{B}$. Then we initialize all lower priority strategies so that $D_{B} \subset B_{1}[t]$ at all further stages $t \geq s$. It follows that $c \in \Theta_{e}^{\Phi_{e}^{A_{1}}, B_{1}} \backslash C_{1}$ and $\mathcal{R}_{e}^{1}$ is satisfied.

If Case (6) never applies for $\alpha$ after stage $s_{1}(\alpha)$ then neither does Cases (8). Hence $\alpha$ does not end prematurely any true stage $t>s_{2}(\alpha)$. Suppose that there is a stage $s_{7}$, such that at all $\alpha$-true stages $t>s_{7}$ Case (7) applies for $\alpha$. It follows that $\Theta_{e}^{\Phi_{e}^{A_{1}}, B_{1}} \neq C_{1}$ and $\mathcal{R}_{e}^{1}$ is satisfied.

Finally suppose that at infinitely many $\alpha$-true stages Case (9) applies. Suppose that $\Theta_{e}^{\Phi_{e}^{A_{1}}, B_{1}}=C_{1}$. We will show that $\Delta_{\alpha}^{\Phi_{e}^{A_{1}}}=G_{1}$. Fix a natural number $n$. If $n \notin G_{1}[t]$ at any stage $t>s_{2}(\alpha)$ then no axiom is ever enumerated in $\Delta_{\alpha}$ for $n$. If $n \in G_{1}$ then $n$ will eventually be assigned final chit $c(n) \in C_{1}$. As $\Theta_{e}^{\Phi_{e}^{A_{1}}, B_{1}}=C_{1}$ there will be a least axiom for $c(n)$ in $\Theta_{e}$ that is valid at all large enough stages. So eventually a valid axiom for $n$ will be enumerated in $\Delta_{\alpha}$.

Suppose that $n \notin G_{1}$ but an axiom $\left\langle n, D_{n} \cup D_{\Phi}\right\rangle$ is enumerated in $\Delta_{\alpha}$ at stage $s$. By Lemma 10 it follows that there is a stage $s_{n}>s$, such that $n \in G_{1}[t]$ at all $t<s_{n}$ and $n \notin G_{1}[t]$ at all $t \geq s_{n}$. We will show that the axiom $\left\langle n, D_{n} \cup D_{\Phi}\right\rangle$ is invalid. The axiom was defined via a chit $c(n)$ and an axiom $\left\langle c(n), D_{\Phi}, D_{B}\right\rangle$ valid at stage $s$ and includes in $D_{n}$ all valid axioms at stage $s$ for elements $n^{\prime}<n$, such that $n^{\prime} \in G_{1}[s]$.

Let $t$ be an $\alpha$-true stage, such that $n \notin G_{1}[t]$. If $B_{1}[s] \uparrow s \subseteq B_{1}[t]$ then $D_{\Phi} \not$ $\Phi_{e}^{A_{1}}[t]$ or else Case (8) would apply to $\alpha$. To complete the proof, we need to show that $B_{1}[s]\left\lceil s \subseteq B_{1}[t]\right.$. Towards a contradiction suppose that $B_{1}[s]\left\lceil s \nsubseteq B_{1}\right.$. Let $\alpha^{\prime}$ be the $\mathcal{R}_{1}$-strategy that changed $B_{1}$ and suppose that this happened at a least stage $t_{0}: s \leq t_{0}<t$. Then at stage $t_{0}$ the strategy $\alpha^{\prime}$ acted under Case (8) and attacked because of some $m \notin G_{1}$. The axiom for $m$ in $\Delta_{\alpha^{\prime}}$ was enumerated at stage $s^{\prime}$, and $\alpha^{\prime}$ extracted a number larger than $s^{\prime}$ defined after $m$ left $G_{1}$. It follows that $s^{\prime}<s$ and $m \notin G_{1}[s]$. Note that if $n \leq d_{\alpha^{\prime}}$ then as we argued above $\alpha^{\prime}$ will define its list $C_{\alpha^{\prime}}$ after stage $s$, and can extract from $B_{1}$ only $\gamma$-markers defined after stage $s$, in particular no number from $D_{B}$. So it follows that $d_{\alpha^{\prime}}<n<s$. By Part (3) of Lemma 9 it follows that if $n \in G_{1}\left[t_{0}\right]$ then $n \in G_{1}$, hence $n \notin G_{1}\left[t_{0}\right]$ and in particular $n \leq m$. The axiom for $m$ in $\Delta_{\alpha^{\prime}}$ is defined before stage $s$ and hence includes an axiom for $n$ which is valid at stage $t_{0}$. As $\alpha^{\prime}$ attacks with the least possible element it follows that $m=n$. But this cannot be, as $m \notin G_{1}[s]$ and $n \in G_{1}[s]$.

To deal with $\mathcal{Q}$ strategies we can use the same approach as in the proof of Theorem 9 .

Lemma 13. Let $\beta$ be a $\mathcal{Q}_{e}$-strategy which is not initialized after stage $s_{0}$ and visited infinitely often. Then $\mathcal{Q}_{e}$ is satisfied and there is a stage $s_{\beta}$ after which $\beta$ does not end stages at which it is visited.

Proof. We first establish the technical fact about how promises are treated. We show that if $\beta$ is a $\mathcal{Q}_{e}$-strategy not initialized in the interval of stages $[s, t]$ and $\beta$ makes a promise $\left\langle D_{X}, D_{G_{1}}, D_{G_{2}}\right\rangle$ at stage $s$, so that at stage $t>s, D_{G_{1}} \oplus D_{G_{2}} \not \subseteq$ $G_{1} \oplus G_{2}$ then there is a stage $t^{\prime} \in(s, t]$, such that $D_{X} \nsubseteq X\left[t^{\prime}\right]$. Suppose for 
concreteness that $o \in D_{G_{1}} \backslash G_{1}[t]$. It follows that $o<s$. First note that $o$ cannot be a threshold of an $\mathcal{R}$-strategy as $\mathcal{R}$-strategies of higher priority do not act and $\mathcal{R}$-strategies of lower priority are initialized at stage $s$. Thus $o$ is an $\Omega$-marker for some number $n \notin X\left[t^{\prime}\right]$ at some stage $t^{\prime} \geq s$. It follows that $i(o)[s]=i(o)[t]=1$ as higher priority strategies do not act in this interval of stages and lower priority strategies are initialized at stage $s$. If $n \notin X[s]$ then by the definition of the $\Omega$ strategy $o \notin G_{1}[s]$. But $D_{G_{1}} \subseteq G_{1}[s]$, hence our assumptions lead to $n \in X[s]$. By the definition of $D_{X}$ it follows that $n \in D_{X}$, thus $D_{X} \not \subset X[t]$.

The rest of the proof can now proceed is that of Lemma 3.

To complete the proof we use Lemma 12 and Lemma 13 to show that the true path exists and use it to prove that the $\Omega$-strategy is successful:

Corollary 3. There is an infinite path $f$ in the tree, such that for every $n, f\lceil n$ is visited infinitely often and initialized only finitely often. All $\mathcal{R}$ and $\mathcal{Q}$ requirements are satisfied.

Lemma 14. $\Omega^{G_{1}, G_{2}}(n)=X(n)$.

Proof. The proof is the same as that of Lemma 8

\section{REFERENCES}

[1] Mingzhong Cai, Hristo A. Ganchev, Steffen Lempp, Joseph S. Miller, and Mariya I. Soskova, Defining totality in the enumeration degrees, submitted.

[2] S. Barry Cooper, Partial degrees and the density problem. Part 2: The enumeration degrees of the $\Sigma_{2}$ sets are dense, J. Symbolic Logic 49 (1984), 503-513.

[3] __ Enumeration reducibilty, nondeterministic computations and relative computability of partial functions, Recursion theory week, Oberwolfach 1989, Lecture notes in mathematics (Heidelberg) (K. Ambos-Spies, G. Muler, and G. E. Sacks, eds.), vol. 1432, Springer-Verlag, 1990, pp. 57-110.

[4] Yu. Ershov, The uppersemilattice of enumerations of a finite set, Alg. Log. 14 (1975), 258284.

[5] Richard M. Friedberg and Hartley Rogers, Jr., Reducibility and completeness for sets of integers, Z. Math. Logik Grundlag. Math. 5 (1959), 117-125.

[6] Hristo Ganchev and Mariya I. Soskova, Cupping and definability in the local structure of the enumeration degrees, J. Symbolic Logic 77 (2012), no. 1, 133-158.

[7] _ Definability via Kalimullin pairs in the structure of the enumeration degrees, Trans. Amer. Math. Soc. (electronic) (2014).

[8] Iskander Sh. Kalimullin, Definability of the jump operator in the enumeration degrees, J. Math. Log. 3 (2003), no. 2, 257-267.

[9] Kevin McEvoy, Jumps of quasi-minimal enumeration degrees, J. Symbolic Logic 50 (1985), 839-848.

[10] Kevin McEvoy and S. Barry Cooper, On minimal pairs of enumeration degrees, Journal of Symbolic Logic 50 (1985), no. 4, 983-1001.

[11] E. Paliutin, Addendum to the paper of Ershov [1975], Alg. Log. 14 (1975), 284-287.

[12] G.E. Sacks, Degrees of unsolvability, Annals of mathematics studies, Princeton University Press, 1963.

[13] Alan L. Selman, Arithmetical reducibilities I, Z. Math. Logik Grundlag. Math. 17 (1971), $335-350$.

[14] J. Shoenfield, On degrees of unsolvability, Ann. of Math. (2) 69 (1959), 644-653.

[15] Stephen G. Simpson, First-order theory of the degrees of recursive unsolvability, Ann. of Math. (2) 105 (1977), no. 1, 121-139.

[16] Theodore A. Slaman and Mariya I. Soskova, The $\Delta_{2}^{0}$ Turing degrees: Automorphisms and definability, submitted.

[17] Theodore A. Slaman and W. Hugh Woodin, Definability in the Turing degrees, Illinois J. Math. 30 (1986), no. 2, 320-334. 
THE ENUMERATION DEGREES: LOCAL AND GLOBAL STRUCTURAL INTERACTIONS 35

[18] _ Definability in the enumeration degrees, Archive for Mathematical Logic 36 (1997), no. 4-5, 255-267 (English).

[19] _ Definability in degree structures, preprint, 2005.

[20] Mariya I. Soskova, The automorphism group of the enumeration degrees, Annals of Pure and Applied Logic, to appear.

(Slaman) Department of Mathematics, University of California, Berkeley, Berkeley, CA 94720-3840, USA

E-mail address, Slaman: slaman@math.berkeley.edu

(Soskova) Faculty of Mathematics and Informatics, Sofia University, 5 James Bourchier Blvd., 1164 Sofia, Bulgaria

E-mail address, Soskova: msoskova@fmi.uni-sofia.bg 